Document downloaded from:

http://hdl.handle.net/10251/159596

This paper must be cited as:

Argoti, MA.; Carrión García, A. (2019). A heuristic method for obtaining quasi ARL-unbiased p-Charts. Quality and Reliability Engineering International. 35(1):47-61.

https://doi.org/10.1002/qre.2379

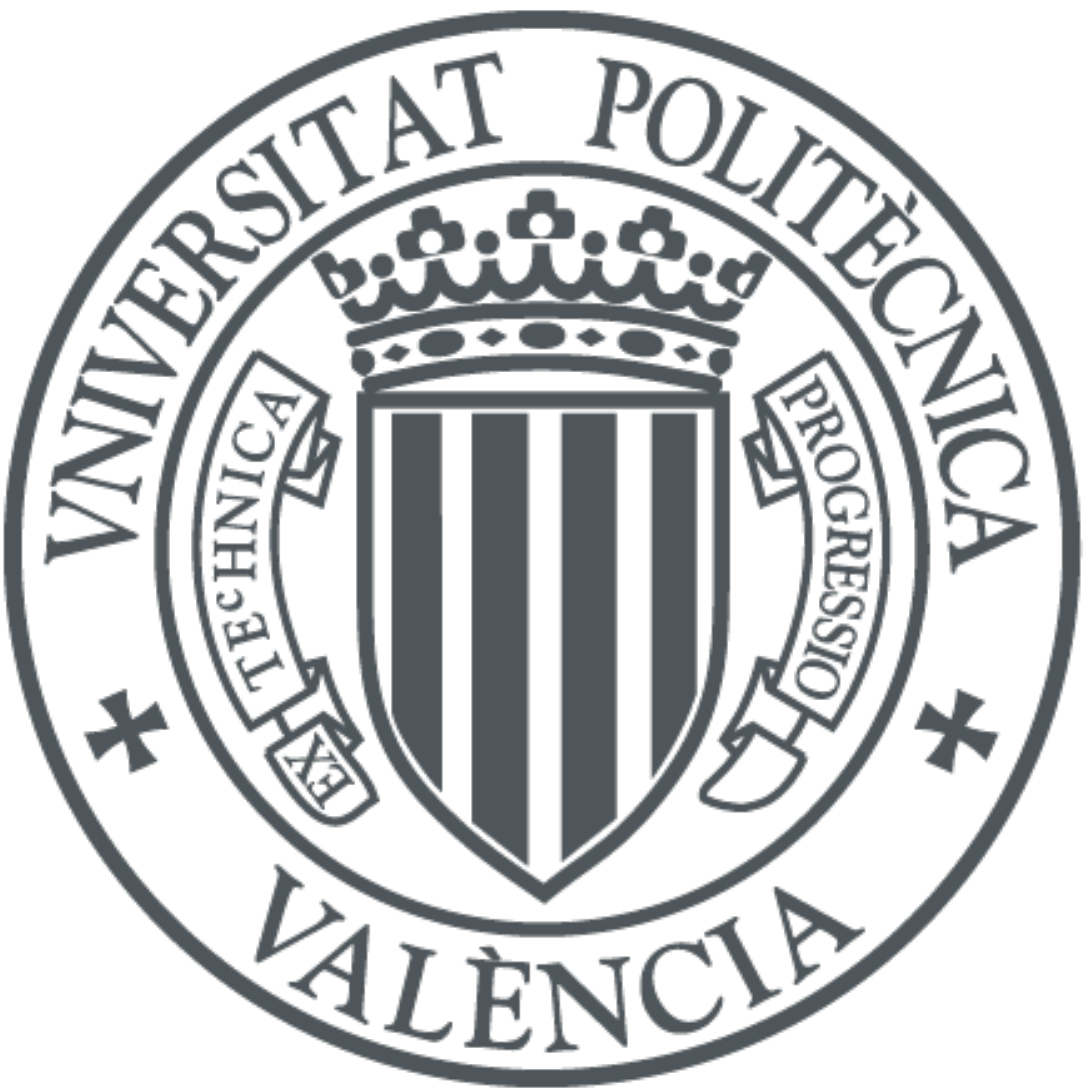

The final publication is available at

https://doi.org/10.1002/qre.2379

Copyright John Wiley \& Sons

Additional Information 


\section{A heuristic method for obtaining quasi ARL-unbiased p-Charts}

Authors:

Marco Antonio Argoti: mararmo@doctor.upv.es

Andrés Carrión García: acarrion@eio.upv.es

Departamento de Estadística e Investigación Operativa Aplicadas y Calidad - DEIOAC

Universitat Politécnica de Valencia

Valencia - Spain

May 2018

\section{Abstract:}

It is known that control charts based on equal tail probability limits are ARL-biased when the distribution of the plotted statistic is skewed. This is the case for $p$-Charts that serve to monitor processes on the basis of the binomial distribution. For the particular case of the standard three sigma Shewhart p-Chart, which is built on the basis of the binomial to normal distribution approximation, this ARL-biased condition is particularly severe and it greatly affects its monitoring capability. Surprisingly, in spite of this, the Standard p-Chart is still widely used and taught. Through a literature search it was identified that several, simple to use, improved alternative p-Charts had been proposed over the years; however, at first instance it wasn't possible to determine which of them was the best. In order to identify the alterative that excelled, an ARL performance comparison was carried out in terms of their ARL bias severity level (ARLBSL) and their In-Control ARL (ARL0). The results indicated that even the best performing alternative charts would often be ARL-biased or have non-optimal ARL0. To improve on the existing alternatives, the "Kmod p-Chart" was developed; it offers easiness of use, superior ARL performance and a simple and effective method for verifying its ARL-bias condition.

Keywords: Attribute control chart; ARL-bias; fraction non-conforming; p-Chart

\section{Introduction}

A widely used tool of statistical process control is the chart that serves to monitor a process by means of its fraction nonconforming, $p$, on the basis of the binomial distribution. Commonly known as $\mathrm{p}$-Chart, its fundamental characteristics are underpinned by the parameters $p$ and sample size, $n$. It is well known that the binomial is a discrete distribution that becomes skewed when $p \neq 0.5$.

In most real applications it is often a requirement for a p-Chart to be able to detect process deterioration and improvement. To achieve this in an optimal way, the following conditions must be achieved: 1) The chart must have upper and lower control limits and 2) The chart's limits must provide probability tail areas that should to be as close as possible to a given nominal value, usually 0.00135 . It is known that $p$-Chart's properties are determined by the reciprocals of its tail areas [1] and that the reciprocal of the combined tail areas is the Average Run Length (ARL), which is the expected number of plotted points before a point falls outside 
the control limits. It is also known that when the tail areas differ in value the chart becomes ARL-biased, an undesired condition that compromises the chart's detection capability [2, p. $189 \& 195]$.

Walter A Shewhart back in the first half of the twentieth century, was the first person to propose a control chart that used $p$. In this paper we called Shewhart's chart the "Stantard p-Chart", its limits are computed by means of equation (1) under the assumption that the binomial distribution is adequately approximated by the normal and that in turn, the chart's limits will provide adequate probability tails areas. This chart, although decades old, is still widely used and taught, Montgomery [3, p. 228] gives a good insight into the theory and applications of this chart. .

Often in real live process monitoring situations the value of $p$ is much lower than 0.5 , this gives rise to a major flaw in the Standard p-Chart in that for small values of $p$, say less than 0.2 , its tail areas are often dissimilar and far-off from the expected nominal value [2, p. 183], even when large sample sizes are used. This is caused by the fact that, for $\mathrm{p}<<0.5$, the normal to binomial approximation is far from accurate, especially on the tail sides [4].

It is rather surprising that the Standard p-Chart, despite its well documented weaknesses, is still extensively used. Factors that may help to explain its enduring popularity could be its simplicity, its easiness of use, the fact that it is relatively easy to figure out its mathematical principals and most probably, unawareness of its deficiencies. Hence, if one is to propose an alternative chart that could become as widely accepted as the Standard p-Chart, apart from having a better performance, it should be just as easy to construct and use.

Several alternative $\mathrm{p}$-Charts and methods that offer control limits that provide tail areas closer to the nominal value have been proposed over the years. Based on the aforesaid alternative chart criteria, we searched and selected only those whose control limits were computed through easyto-use methods. Grouped according to the approach used to derive them, hereunder we mention those we consider fulfil the criteria appropriately:

a) Optimum control limits approach: Under this approach are the charts that use a closedform equation to compute control limits that provide improved probability tails Examples of these charts are: 1) The chart based on a rule of thumb correction proposed by Ryan [5, p. 348].The chart based on the Corner-Fisher expansion originally proposed by Winterbottom [6] and later extended by Chen [7] 3) The chart based on regression analysis proposed by Ryan\&Schwertman [1] and 4) The chart based on the Wilson interval proposed by Park [8].

b) Transformation approach: Under this approach are the charts that utilize a normalizing transformation to obtain improved probability tails. Examples of these are: 1) the Arcsine Chart [2, p. 188] and 2) The Q Chart [9], [10].

As can be seen several simple to use alternatives to the Standard p-Chart have been proposed over the years. However, once the list of viable alternatives had been drawn, it became clear that two important questions had to be answered, these were: which of them is the better alternative? And, could a new viable alternative chart be developed? The search for answers to these questions led us to carry out the research summarised in this paper.

In order to identify the alternative chart that excelled, a performance comparison in terms of their ARL performance was carried out; for this we developed a new parameter called ARLbias severity level (ARLBSL), this parameter along with the In-Control ARL made it possible to objectively compare all of the charts. Employing the knowledge gained throughout the research, 
a new easy-to-use "closed-form equation" type chart was developed; we called it "Kmod pChart", it offers easiness of use, excellent ARL performance for a wide range of $p$ values and a simple method for verifying its ARL-bias condition.

This paper is organized as follows: Section 1 contains firstly a short description of the Standard p-Chart followed by a brief analysis of the binomial distribution, then a description of the ARLBSL and finally a brief summary of the ARL performance for the Standard p-Chart. In Section 2, firstly we present the Kmod p-Chart and its ARL performance, followed by a graphical description of binomial tail performances for selected alternative charts and then finally we present a table that allows comparing the ARL performance of several alternative pCharts. Section 3 contains concluding remarks.

\section{Materials y method}

\subsection{The Standard p-Chart}

Let's suppose that we have items made in a stable production process out of which independent samples, of size $n$, are inspected and that the number of nonconforming items in the $i$-th sample is given by the Bernoulli parameter $p$. In this case the number of nonconforming items obtained in each sample, denoted as $x_{i}(i=1,2,3, \ldots)$, could be modelled as a random variable that follows a binomial probability distribution.

If $p$ is known and if $n$ is sufficiently large then the process could be statistically monitored by plotting the fraction of nonconforming $y_{i}=x_{i} / n$ on a Standard $p$-Chart with limits given by equation (1). This equation is derived using the Central Limit Theorem (CLT) under the assumption that the binomial distribution is approximately normal.

$$
\text { Control Limits }=p \pm K \sqrt{\frac{p(1-p)}{n}}
$$

Where:

- For upper control limit (UCL) use +

- For lower control limit (LCL) use -

- $\mathrm{K}=\mathrm{Z}_{(1-\alpha / 2)}$

- $\alpha=$ Type I error probability (or Alpha error), $\mathrm{n}=$ sample size and $\mathrm{p}=$ Known process fraction of nonconforming.

Often in real practical situations the value of $p$ is not known and consequently it must be estimated. In this case the maximum likelihood estimator of $p$ must be calculated as shown in (2).

$$
\bar{p}=\frac{\sum_{i=1}^{m} x_{i}}{N}
$$

Where: $N=\sum_{i=1}^{m} n_{i}$

$$
\begin{aligned}
& n_{i}(i=1,2, \ldots \ldots m) \rightarrow \text { A predefined group of } m \text { randomly selected samples of size } n_{i} \\
& \qquad \text { In our case, } n_{i}=n \forall i \\
& x_{i}=\text { Count of nonconforming items in the } i \text {-th sample }
\end{aligned}
$$

In (1) $\mathrm{K}$ is the $(1-\alpha / 2)$ th quantile of the standard normal distribution $N(0,1)$. To obtain the widely used three sigma $\mathrm{p}$-Chart a false alarm rate, or alpha error probability, value of $\alpha=$ 
0.0027 is used. This means that when $\mathrm{K}=3$ and the process is In-Control (or IC), the lower and upper alpha tail error probabilities should be equal to $\alpha / 2$ or 0.00135 . In this paper we call these tail values $I C$ alpha tails. Notice that these tail values are obtained assuming the binomial distribution to be approximately normal, which would consequently imply that its distribution shape is almost symmetric or non-skewed.

The IC alpha tails play a determinant role on a p-Chart's monitoring capability, hence their true value ought to be determined if one is considering using this type of chart. The tails can be calculated by means of equations (3) and (4). These equations are incorporated in widely used computer software programs, like Excel's binomial probability function; hence, the IC alpha tails can be easily computed without the need of doing the calculations by hand.

$\alpha_{U}=$ Upper IC alpha tail

$\alpha_{U}=P\left\{x \geq\left. n U C L\right|_{p}\right\}$

$\alpha_{U}=1-\sum_{0}^{n U C L}\left(\begin{array}{l}n \\ x\end{array}\right) p^{x}(1-p)^{n-x} \quad x=0,1,2 \ldots n U C L$

$\alpha_{L}=$ Lower IC alpha tail

$\alpha_{L}=P\left\{x \leq\left. n L C L\right|_{p}\right\}$

$\alpha_{L}=\sum_{0}^{n L C L}\left(\begin{array}{l}n \\ x\end{array}\right) p^{x}(1-p)^{n-x} \quad x=0,1,2 \ldots n L C L$

Also:

$\alpha_{o}=\alpha_{L}+\alpha_{U}=$ Total IC alpha error probability

In this point we also define the IC alpha tails ratio as:

$\mathrm{R}_{\alpha}=\alpha_{\mathrm{L}} / \alpha_{\mathrm{U}}$

Since the lower and the upper tail values should, in theory, be equal then the expected IC alpha tails ratio is $\mathrm{R}_{\alpha}=1$.

\subsubsection{The Average Run Length - ARL}

When monitoring a process with a $\mathrm{p}$-Chart, there will be points $y_{i}$ that fall inside and outside the chart limits. The average number of $y_{i}$ points that fall inside the chart limits before one $y_{i}$ falls outside them is called the Average Run Length (ARL), see [3, p. 191] \& [11, p. 277]. The ARL is commonly used to measure the performance of a control chart, and it can be classified in two types, namely: i) The In-Control ARL, usually identified as ARL 0 and ii) The Out of Control ARL identified in this paper as ARL1.

The ARL 0 exists when the monitored process is working under In-Control (or IC) conditions. In IC conditions the $y_{i}$ points that fall outside the chart limits do so according to the false alarm rate at which the chart has been designed, a typical alarm rate is $1 / 370=0.0027$, hence in this case the expected $\mathrm{ARL} 0=1 / 0.0027 \approx 370$. When the average $y_{i}$ points that fall outside the limits do so at a higher rate than the ARLo, it is an indication that the process is out of control (or $O C$ ). The following situations could cause an OC state: 1) An increment in the fraction of nonconforming (or positive p-shift), that may be caused by process deterioration and 2) A decrease in the fraction of nonconforming (or negative $\mathrm{p}$-shift), that may be caused by a process improvement.

A chart's capability to detect an OC state is commonly measured by means of the ARL $\mathrm{A}_{1}$, that being the average number of $y_{i}$ points plotted within the limits before a $y_{i}$ point appears 
outside them when the process fraction of nonconforming, $p$, has suffered a positive or negative shift. In this paper we denote a p-shift as $\mathrm{p}_{1}$.

\subsection{Skewness and discreetness of the binomial distribution and its effect on the p-Chart}

It is well known that the shape of the binomial distribution is directly related to its parameters $p$ and $n$ and that only when $\mathrm{p}=0.5$ its shape is symmetric for large or small $n$ values. When $\mathrm{p}<0.5$ and $n$ is small, the distribution becomes right skewed, however, when $n$ is sufficiently large the skewness reduces to the point that it becomes nearly symmetric, when this happens the Central Limit Theorem (or CLT) could be used to successfully approximate the binomial to the normal distribution. A common rule of thumb often found in text books says that the approximation would be adequate so long as $n p \geq 5$ (or 10); thus one could assume that a process could be satisfactorily monitored by a p-Chart provided that the conditions of this rule are met.

As previously shown, a p-Chart is built under the assumption that its control limits will provide IC alpha tails equal to 0.00135 . However, the equal tail value condition is not achieved even when $n p>10$. To illustrate why this happens we present Figure 1, it shows the binomial density distribution for $\mathrm{n}=244$ and $\mathrm{p}=0.05$ and the shape of its CLT normal approximation; the $n$ and $p$ combination gives an $n p=12.2$. Notice that the binomial probability density has discrete values whose distribution is slightly right skewed and that the normal approximation does not accurately follow its shape. Most importantly notice how the tails of the two distributions differ, the normal approximation lower tail runs above the binomial whilst its upper tail runs below it. The $\mathrm{p}$-Chart's control limits for this $\mathrm{n}$ and $p$ combination, identified as nLCL and nUCL, denote the boundaries under the normal curve where the probability areas are approximately equal to 0.00135 . However, due to the inaccurate fit of the binomial to the normal, the locations of these limits provide IC alpha tails that are rather different from the expected 0.00135 . This problem is made worse by the inherent binomial discreteness, as will be explained next.

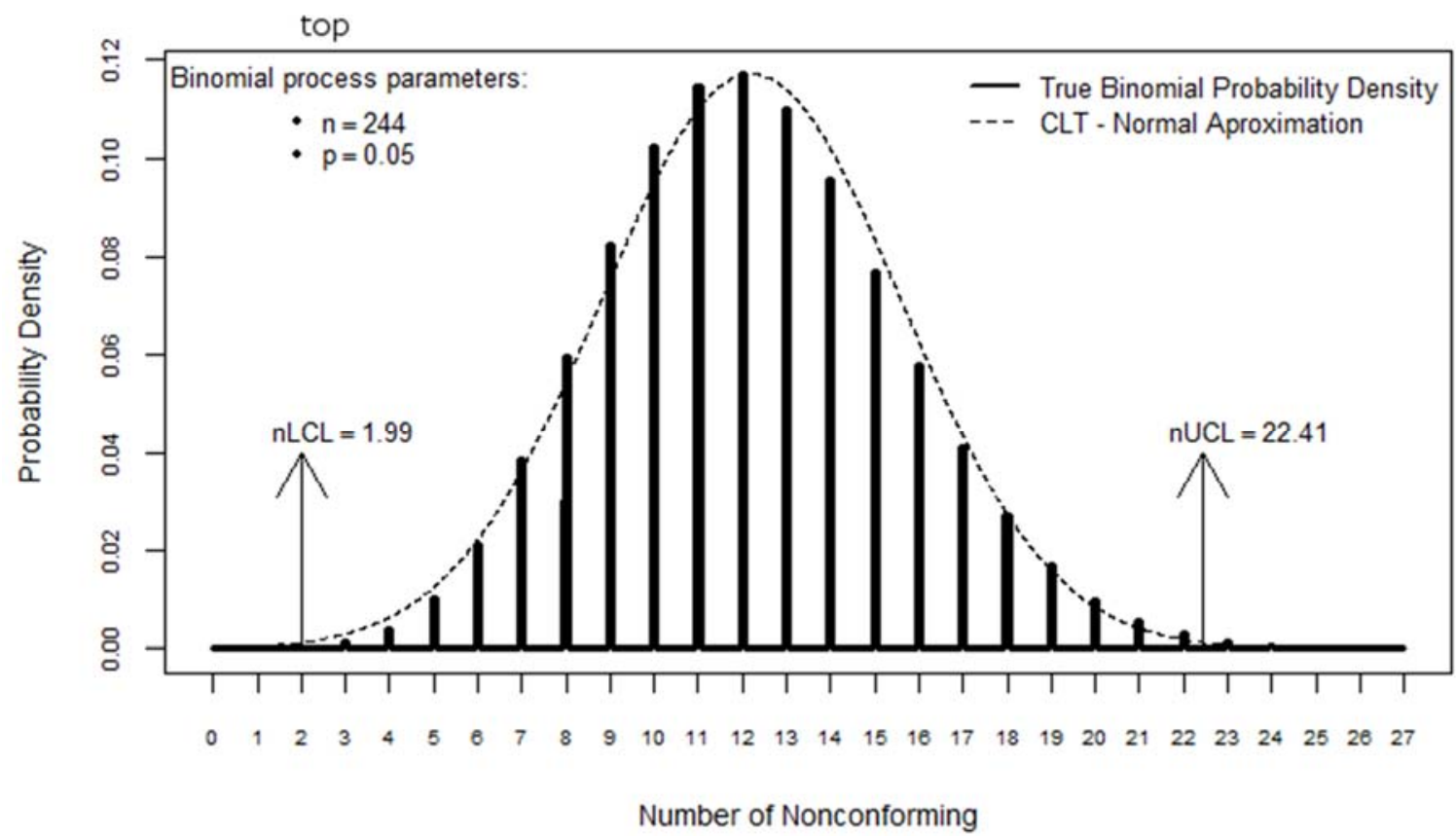

Figure 1: Example of the discrepancy between the binomial distribution and its normal approximation 
To illustrate in more detail the effect that the binomial skewness and discreetness has on the IC alpha tails we present Figure 2 and Figure 3. Figure 2 shows the upper and lower tails of the cumulative binomial probability curve for $n=244$ and $p=0.05$. Notice that the probabilities values are discrete and that only in the upper tail, between 23 and 23.99, there is a discrete value equal to 0.00135 . The lower tail value obtained with $\mathrm{nLCL}=1.99$ is 0.000051 , which is 26.5 times below 0.00135 . In the other hand with $\mathrm{nUCL}=22.41$ the tail obtained is 0.00288 , which is 2.13 times above 0.00135 . Overall we have that the alpha tails ratio, as defined in (6), is $\mathrm{R}_{\alpha}=0.017$; which denotes a substantial difference between the lower and upper alpha tails.

Figure 3 shows the tails of the cumulative binomial probability curve for and $n=245$ and $p=0.05$, this sample size is just one above to the one used in Figure 2. Notice that in this case in neither tail exist a probability value that would provide the expected 0.00135 , only in the upper tail the discrete value between 23 and 23.99 comes close to it. The lower tail value obtained with $\mathrm{nLCL}=2.02$ is 0.000337 , which is 4 times below 0.00135 , whilst the upper tail value obtained with $\mathrm{nUCL}=22.48$ is 0.00303 , which is 2.24 times above 0.00135 . The alpha tails ratio in this case is $R_{\alpha}=0.11$ which in comparison to the one obtained for $n=244$ is about 6.5 times bigger. This clearly demonstrates that just a small change in the sample size can cause a huge variation in the IC alpha tails.

Figure 2 and Figure 3 served to demonstrate that the inaccurate fit of the normal to the binomial distribution causes the IC alpha tails to be: i) much below 0.00135 in the lower tail and ii) much above 0.00135 in the upper tail. The figures also helped to show that due to the binomial distribution discreetness, substantial variations can occur in the IC alpha tails with just a small change in the binomial parameter $n$.

Notice that an $\mathrm{R}_{\alpha}$ closer to one could have been obtained in both examples, if the control limits could have been shifted to values that provide alpha tails nearer to 0.00135 .

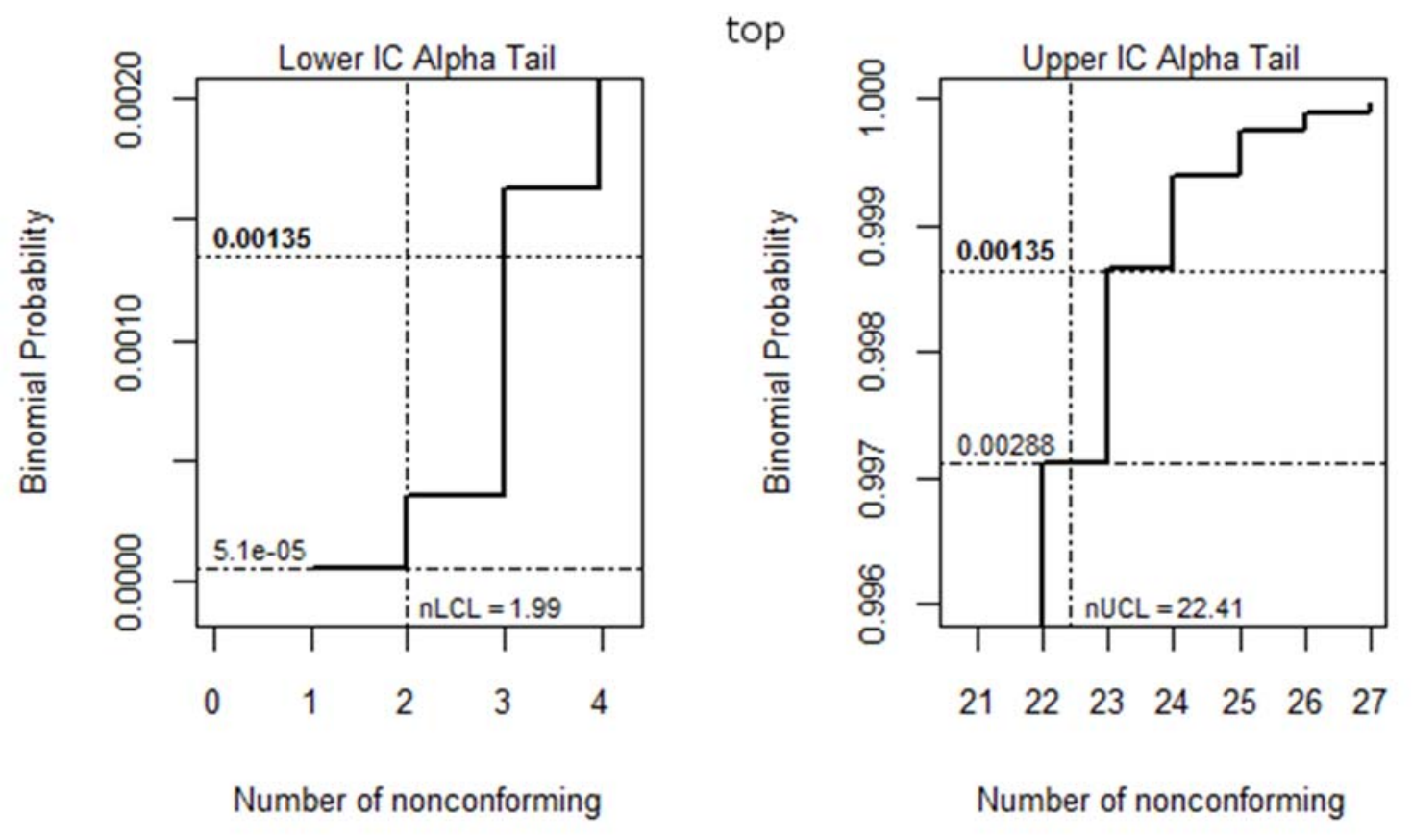

Figure 2: Upper and lower tails of the cumulative binomial probability for $\mathrm{p}=0.05$ and $\mathrm{n}=244$ 


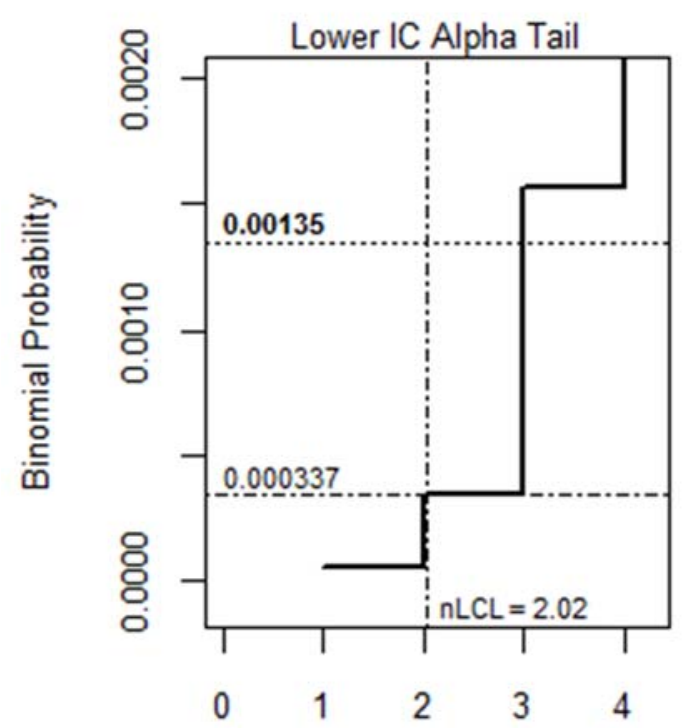

Number of nonconforming

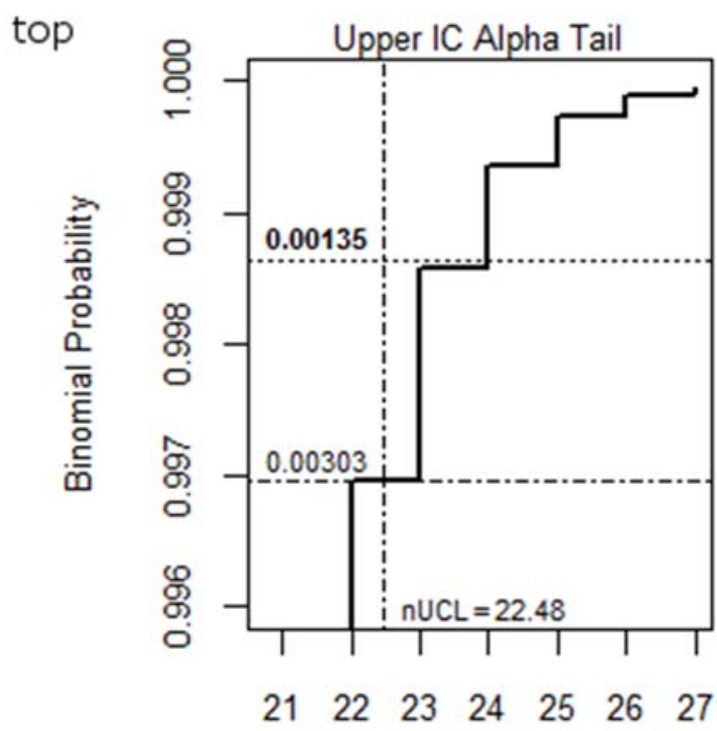

Number of nonconforming

Figure 3: Upper and lower tails of the cumulative binomial probability for $\mathrm{p}=0.05$ and $\mathrm{n}=245$

\subsubsection{The Standart p-Chart IC alpha tails oscillation}

In order to determine how the IC alpha tails behaves for different $p$ and $n$ combinations, we developed an algorithm capable of computing the tail values for any $p$ and $n$ combination and used it to conduct an analysis between $p \geq 0.01$ and $p \leq 0.2$. The sample size range used for the analysis was specific to each $p$ and was obtained using (7); this equation is given by Duncan $[12$, p. 451] and it is commonly used to obtain a p-Chart's sample size.

The sample size range minimum $n$ was obtained setting $\mathrm{d}=0.99$, this value was chosen due to the fact that it provides the approximate minimum $n$ required by a Standard $\mathrm{p}$-Chart to have a LCL. For the maximum $n$ we set $\mathrm{d}=\mathrm{p} / 3$.

$$
\text { sample size }(n)=9 \mathrm{p}(1-\mathrm{p}) / d^{2}
$$

As an example of the results we present Figure 4. This figure display the IC alpha tails in function of $n$ for $p=0.05$. As can be seen, the upper tails oscillate above 0.00135 whilst the lower tails oscillate below it. At low $n$ values the tails get further apart with the lower tail getting closer to zero, whilst at higher $n$ the tails get closer converging towards, but never reaching, the expected 0.00135 value. We found this behaviour to be similar for all other $p$ values used in the study. Notice also that even at high sample sizes, where values are substantially above the rule of thumb $\mathrm{np}>10$, the IC alpha tails fail to reach the expected value; this clearly demonstrates that this rule of thumb simply does not provide satisfactory results when applied to $\mathrm{p}$-Charts.

Ryan [2, p. 190] shows a table that includes the actual IC alpha tails values for several $n$ and $p$ combinations and uses this to conclude that the alpha tails for the three sigma Standard p-Chart, deviate from the expected 0.00135 value quite substantially. Our results confirms, albeit in a different way, Ryan's conclusion regarding the Standard p-Chart IC alpha tails. 


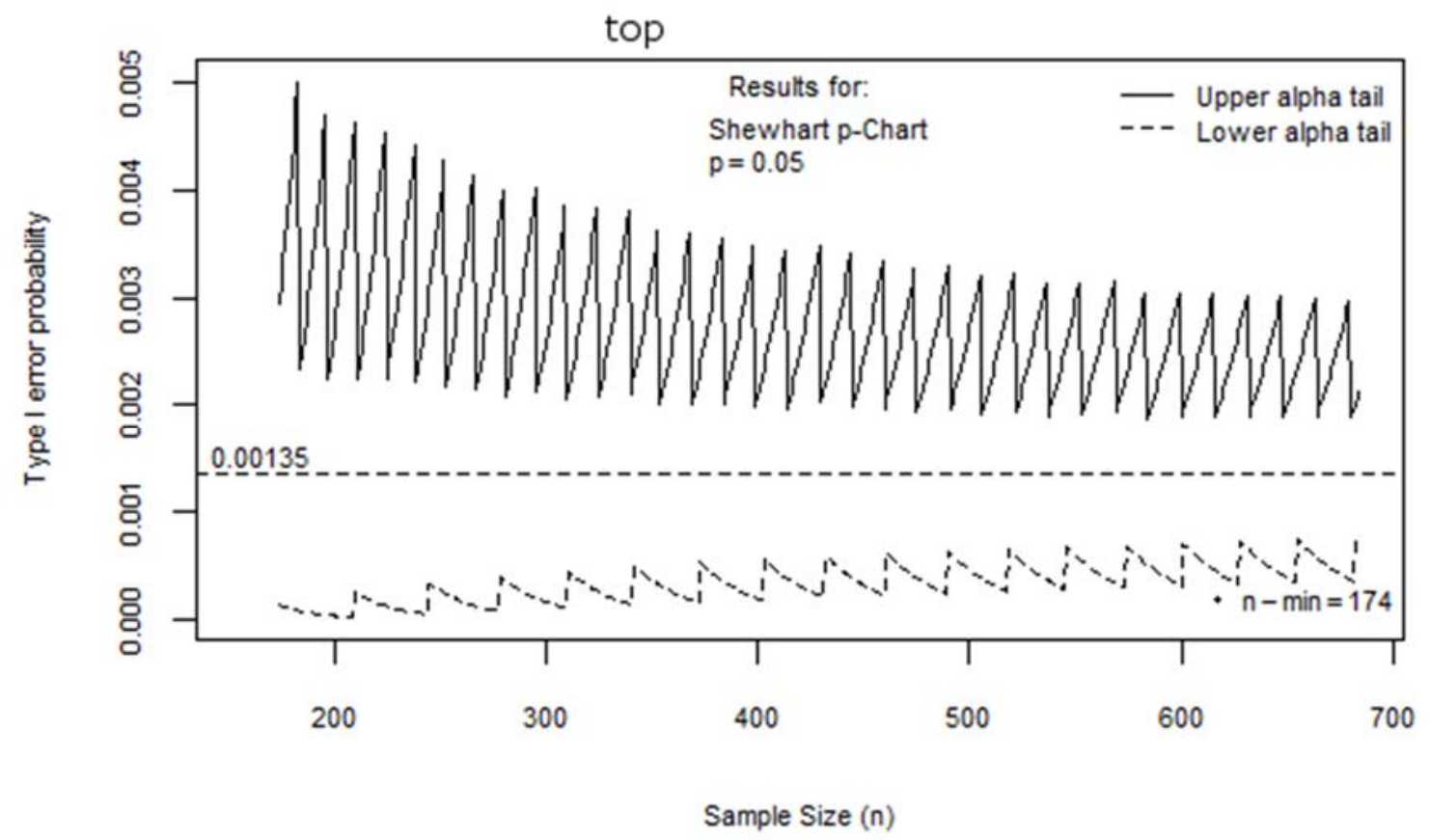

Figure 4: Example of Standard p-Chart's typical IC alpha tails behaviour for fixed $p$ and variable $n$

\subsubsection{The IC alpha tails and the In-Control Average Run Length - ARL}

As previously mentioned a chart's ARL can be classified into two types, ARLo and ARL1. Let's recall that the In-Control ARL, or ARL0, is the average number of points that fall within the chart limits before a point fall outside them when the process is In-Control (or IC). In this case, giving that the process is IC, the points that fall outside the limits are considered to be false alarms.

The ARL 0 is a function of the IC alpha tails and can be calculated by means of equation (8).

$$
A R L_{0}=\frac{1}{\alpha_{o}}
$$

Where:

$$
\begin{aligned}
& \alpha_{o}=\alpha_{L}+\alpha_{U}=\text { Total IC alpha error probability } \\
& \alpha_{U}=\text { IC upper alpha tail } \\
& \alpha_{L}=\text { IC lower alpha tail }
\end{aligned}
$$

As previously shown a three sigma Standard p-Chart should have, in theory, IC alpha tail values equal to 0.00135 , using (8) we obtain that in this case the expected ARL $\mathrm{A}_{0}$ is: $1 /\left(\alpha_{L}+\alpha_{U}\right)=$ $1 /(0.00135+0.00135)=1 /(0.0027) \approx 370$. This means that when the process is working under IC conditions, in average there would be one false alarm, or one point erroneously plotted outside the chart limits, every 370 samples. However, since the ARL 0 is a function of the IC alpha tails and these oscillate, then its value will also oscillate. As an example we present Figure 5 , it shows the corresponding ARL 0 for the alpha tails of Figure 4. Notice that often the ARL 0 is below 370, in those cases there will be more false alarms than expected, giving the erroneous impression that the process is out of control. We found this behaviour to be similar for all the other $p$ included in our analysis. 


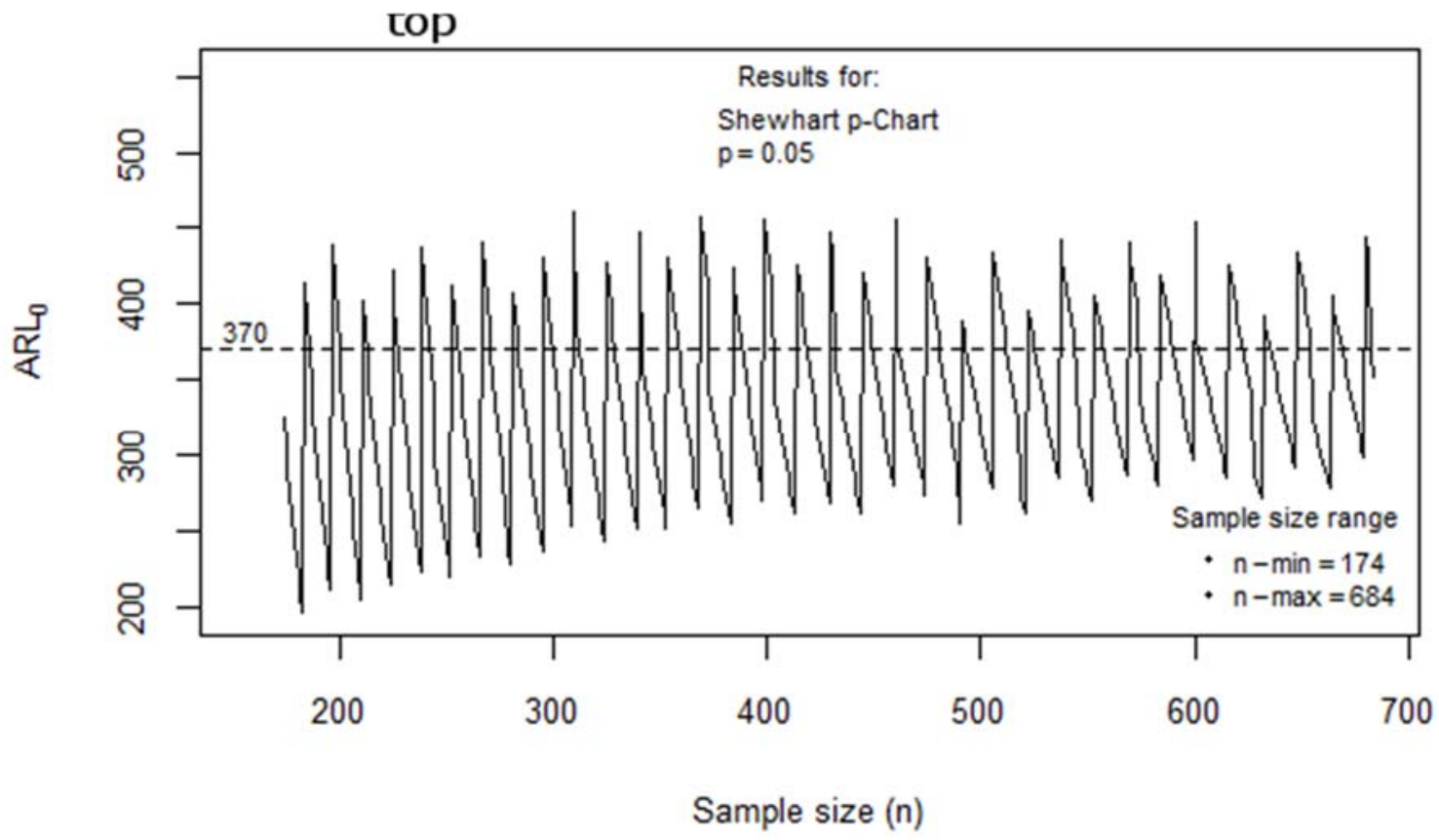

Figure 5: Example of the oscillation of the $\mathrm{ARL}_{0}$ on the Standard p-Charts

\subsection{The p-Chart's ARL Curve}

\subsubsection{Computing the Out of Control Average Run Length - ARL 1}

As mentioned before a process could be Out of Control (or OC) due to positive or negative shifts in the process fraction of nonconforming, $p$, in this paper we denote these shifts as $p_{l}$. The ARL 1 , or average number of points that fall inside the chart when there has been a p-shift, is computed by means of equations (9) and (10).

$$
\begin{gathered}
A R L_{1}=\frac{1}{1-\beta} \\
\beta=P\left\{x \leq\left. n U C L\right|_{p=p_{1}}\right\}-P\left\{x \leq\left. n L C L\right|_{p=p_{1}}\right\}
\end{gathered}
$$

Where:

$$
\begin{aligned}
& p_{1}=p \text {-shift } \quad * p_{1} \text { could be: } p_{1}<\mathrm{u} \text { or } p_{1}>\mathrm{u} \\
& x=\text { number of nonconforming units } \\
& \beta=\text { Type II error probability }
\end{aligned}
$$

\subsubsection{The ARL curve}

In order to analyse a p-Chart's capability to detect an OC state, one could generate $\mathrm{p}_{1}$ values above and below $p$ and then compute their corresponding ARL1. By plotting the results, the chart's ARL curve is obtained. Figure 6 shows an ideal ARL curve, we've chosen to present the $\mathrm{x}$-axis as the percentual change $\left(p_{1} / p\right)-1$, where $p$ is the process fraction of nonconforming and $p_{1}$ denotes the actual $\mathrm{p}$-shift. In this case $\mathrm{p}_{1}=\delta p$, with $\delta$ being the relative shift coefficient that can take values above or below one. For example: if $p=0.1$ and $\delta=0.9$ the resulting $\mathrm{p}_{1}$ is 0.09 and hence $\left(p_{1} / p\right)-1=-0.1$, denoting that the process fraction of nonconforming has suffered a $10 \%$ negative shift. 


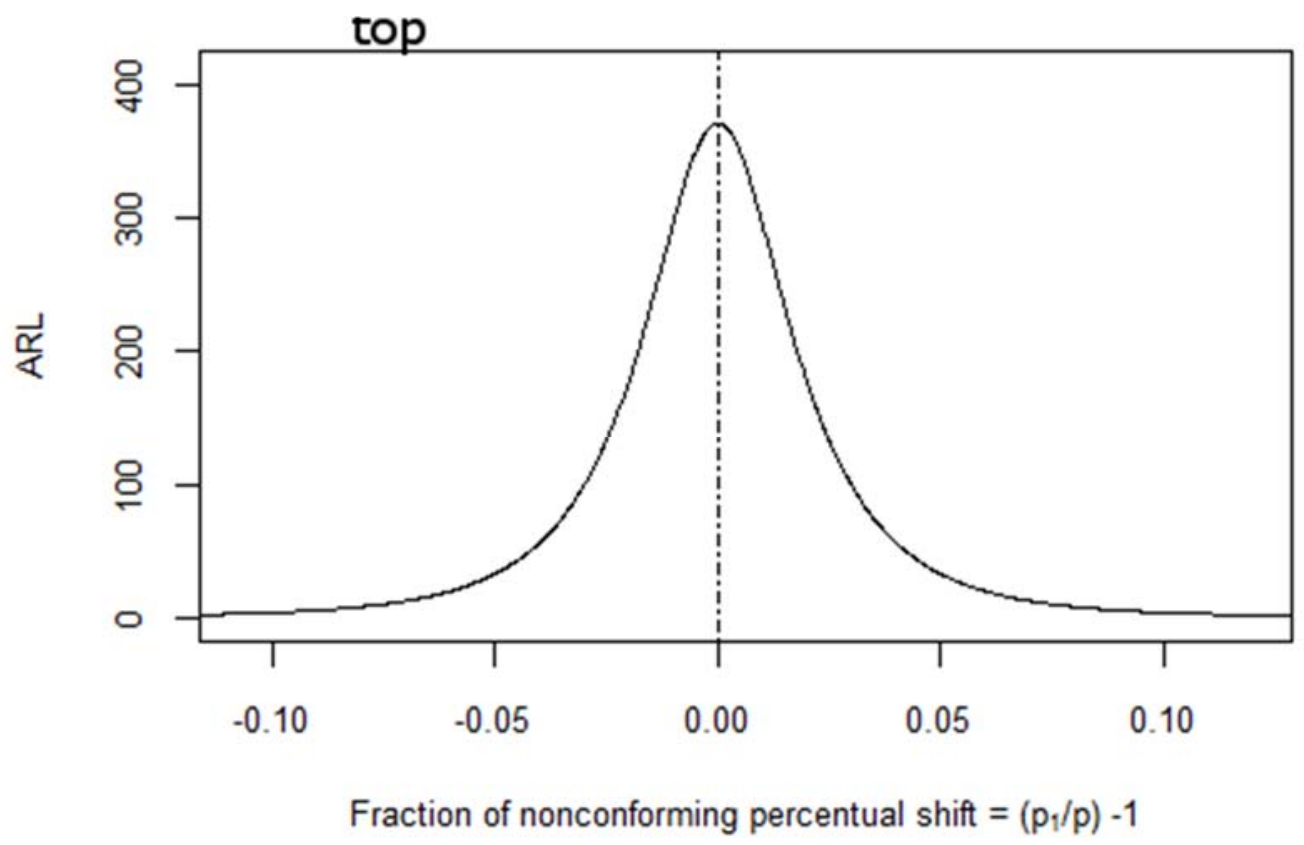

Figure 6: Example of unbiased ARL curve

In Figure 6 the $\mathrm{x}$-axis point $\left(p_{1} / p\right)-1=0$ indicates that there isn't a p-shift, hence its corresponding ARL value is the $\operatorname{ARL}$. When $\left(p_{1} / p\right)-1 \neq 0$ there is a p-shift and its corresponding ARL is the OC-ARL (or $\mathrm{ARL}_{1}$ ). It can be seen that always $\mathrm{ARL}_{1}<\mathrm{ARL}_{0}$ and that the curve is symmetric with respect to the y-axis. A p-Chart that has this type of ARL curve will have equal capability to detect process deterioration or improvement.

The ARL curve shown in Figure 6 is also known as an unbiased ARL curve. Acosta-Mejia [13] defined a control chart as being ARL-unbiased if its ARL curve decreased as the process parameter moved away from the in-control value, for a $\mathrm{p}$-Chart the process parameter is $p$.

\subsubsection{The biased ARL curve and the ARL-bias severity level}

Figure 7 shows the ARL curves for the $p$ and $n$ combinations used for Figures 2 and 3. Both curves are biased towards the negative $\mathrm{p}$-shift direction, in this paper we call this type of bias "negative ARL-bias", when the bias is towards the positive p-shift we call it "positive ARLbias". The curves indicate that with negative ARL-bias, the ARL 1 required to detect a certain range of negative $p$-shifts will be higher than the ARL 0 . It is easy to deduce that $p$-Charts with these ARL characteristics would have a diminished process improvement detection capability.

Notice how both curves in Figure 7 vary in height and width indicating a very different ARLbias severity despite the fact that the only difference in their binomial parameters, $p$ and $n$, is just one sample size unit, from 244 to 245 . Notice also that in both curves the ARL peak value (ARLM) is higher than the ARL 0 and that the $\mathrm{x}$-axis location of this peak is different to that of the ARLo. The variations of the ARLM relative to the ARL 0 can be used to quantify a p-Chart's ARL-bias severity level.

In this paper we use the following parameters to quantify the ARL-bias severity level (or ARLBSL):

1) $\quad$ The ARL-ratio $=$ ARLm $/ A R L 0$

Where:

- $\quad \mathrm{ARL}=\mathrm{ARL}$ curve peak value

- $\mathrm{ARL}_{0}=\mathrm{In}-$ Control ARL 
Ideally $\left(\mathrm{ARL}_{\mathrm{M}} / \mathrm{ARL}_{0}\right)=1$, otherwise the higher the ratio the greater the bias severity level.

Something that must be considered is that excessively low, or high, $\mathrm{ARL}_{0}$ values may be undesirable for process monitoring [14, p. 80]. We considered an ARLo to be acceptable if it falls within the range of 250 and 450 .

2) The relative ARL-bias, denoted in this paper as ARL-bias(\%).

$$
\operatorname{ARL}-\operatorname{bias}(\%)=100 *\left[\left(p_{M} / p\right)-1\right]
$$

Where $\mathrm{p}_{M}$ is the $\mathrm{p}$-shift at which the ARL $L_{M}$ happens.

The relative ARL-bias can be seen as the percentage difference between the location of the ARLM and the ARL 0 in the x-axis. Ideally the ARL-bias(\%) should be equal to zero, otherwise the further apart from zero the greater the severity level. A negative sign before the ARL-bias(\%) value indicates a negative ARL-bias.

An actual value for the ARLBSL can be obtained using equation (11). Given that by multiplying the ideal values of the ARL-ratio and the ARL-bias(\%) we obtain zero, then the ideal ARL-bias severity level should also be zero. An ARLBSL $=0$ indicates that the ARL curve is unbiased, the more severe the ARL-bias the further the ARLBSL departs from zero. A negative sign denotes a negative ARL-bias whilst a positive denotes a positive ARL-bias.

$A R L$-bias severity level $\left(A R L_{B S L}\right)=A R L$-ratio $* A R L$-bias $(\%)$

Figure 7 includes the ARLBSL values of each ARL curve and also their IC alpha tails ratio $\mathrm{R}_{\alpha}$. Notice how the curve with the $\mathrm{R}_{\alpha}$ value closest to one has the lowest ARLBSL, this is an example of the improvement that can be gained in the ARLBSL by making the IC alpha tails closer to 1 .

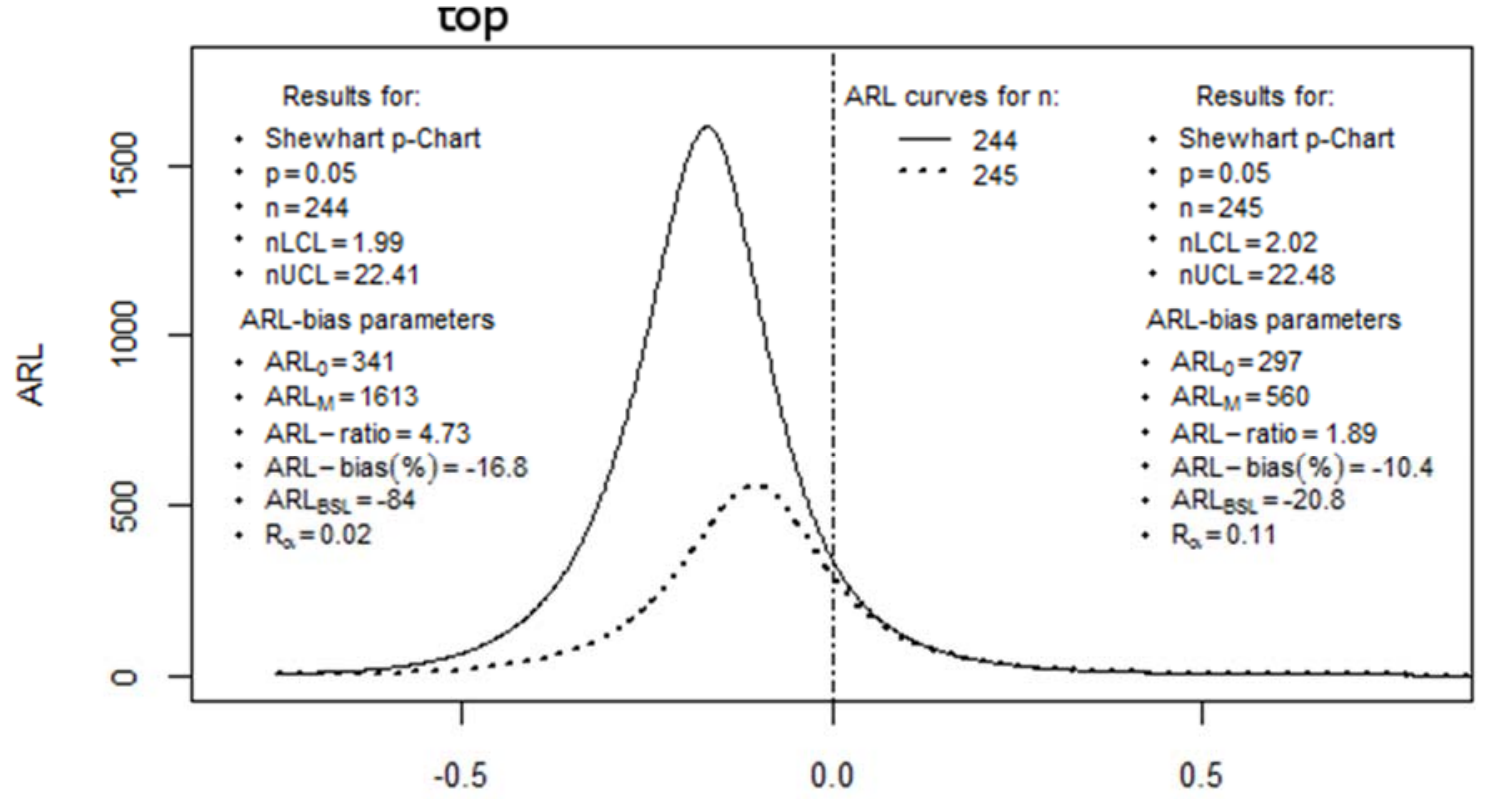

Fraction of nonconforming percentual shift $=\left(p_{1} / p\right)-1$

Figure 7: Example of biased ARL curves 


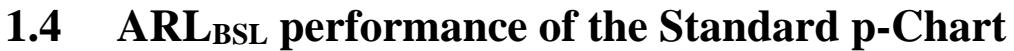

In order to ascertain the ARL-bias severity behaviour of ARL curves produced by different combinations of $p$ and $n$, we made an algorithm capable of computing the following parameters: i) The ARL-ratio, ii) The ARL-bias(\%) and iii) The ARLBSL. This algorithm was used to undertake an extensive Standard p-Chart's ARL-bias severity study, that included fractions of nonconforming between $p \geq 0.01$ and $p \leq 0.2$ and sample sizes ranges specific for each $p$ that were determined following the same criteria used in section 1.2.1.

Typical examples of the results obtained for the ARL-ratio and the ARL-bias(\%) are presented in Figures 8, and 9. For reasons of conciseness we present results only for $p=0.05$. As can be seen the ARL-ratio oscillates in a logarithmically decreasing manner, approaching the desired value of one at very high $n$ values. In the other hand, the ARL-bias(\%) also oscillates but in an logarithmically increasing manner approaching the desired value of zero at very high sample sizes.

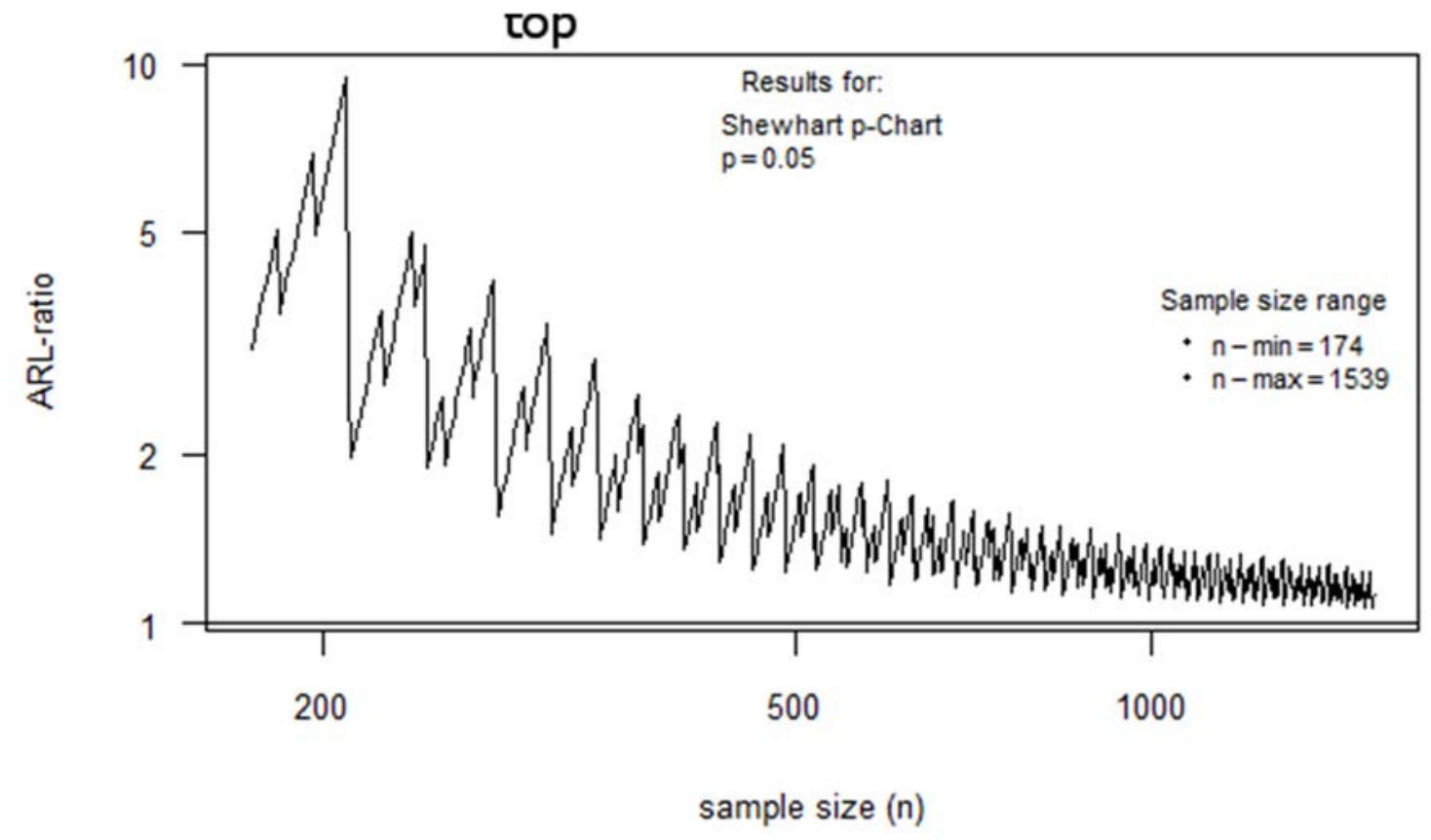

Figure 8: Example of the ARL-ratio in function of sample size and fixed $p$ 


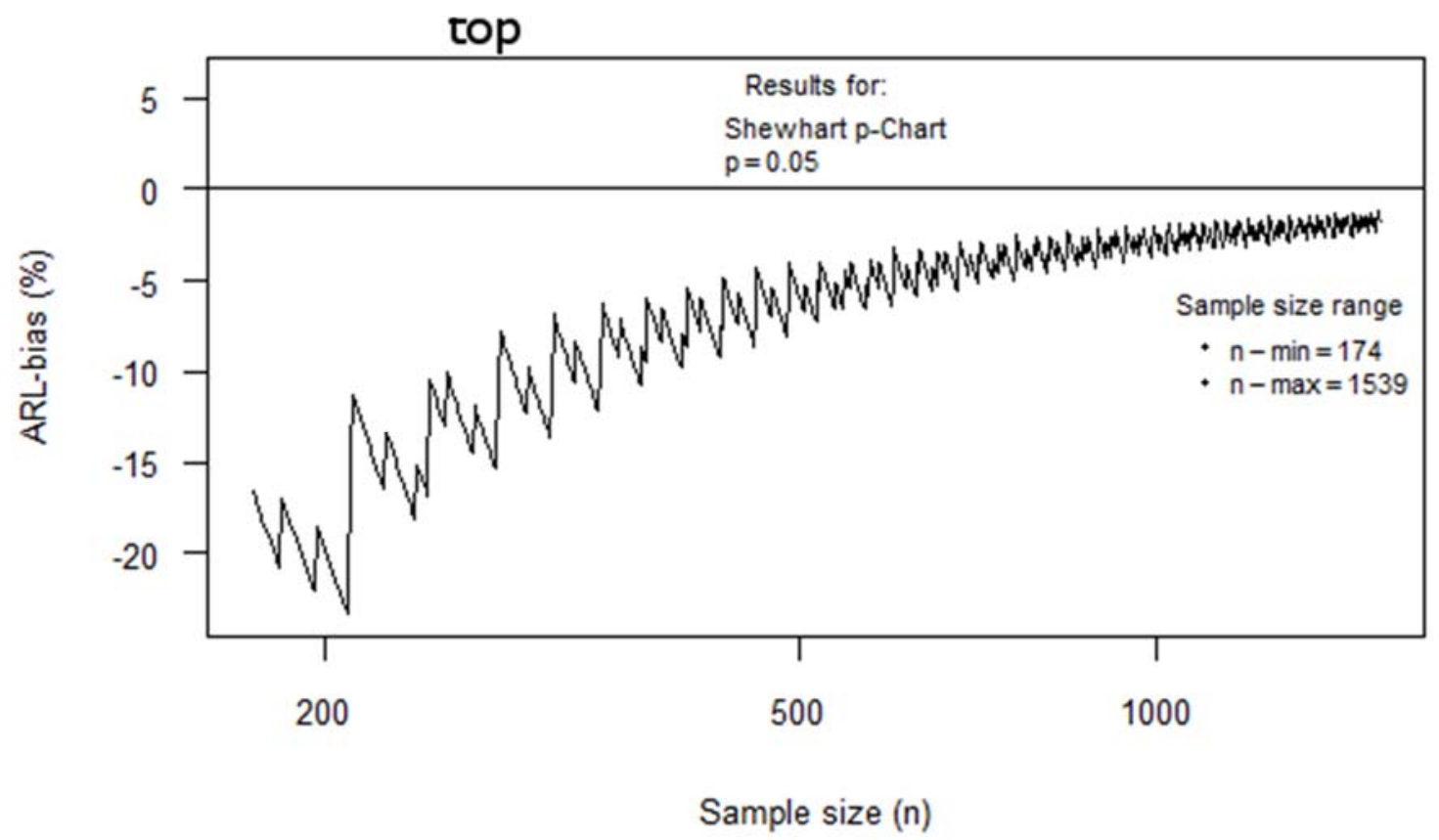

Figure 9: Example of the relative ARL-bias in function of sample size and fixed $p$

After an in-depth analysis of the overall ARL-ratio and ARL-bias(\%) results, and also after careful consideration, we reached the conclusion that ARL-ratios below 1.1 combined with ARL-bias(\%) within \pm 2 would produce control charts with an ARL-bias that could be considered to have negligible effect on the chart's p-shift detection capability. In this paper we call these charts "quasi ARL-unbiased". In terms of the ARLBSL, we considered a p-Chart to be "quasi ARL-unbiased" if it falls within the range: $-2<\mathrm{ARL}_{\mathrm{BSL}}<2$.

Figure 10 depicts typical Standard p-Chart's ARLBSL behaviour for fixed $p$ and variable sample size. As can be seen, the ARLBSL increases negatively as $n$ reduces in size and only at the extreme higher end a handful of $n$ values achieve the ARLBSL $>-2$ criterion; very similar ARLBSL behaviour was found for all other $p$ used in our study. Figure 10, and also Figure 11; help to demonstrate that for $\mathrm{p}<<0.2$, the vast majority of Standard $\mathrm{p}$-Charts will have negative ARL-bias with severity levels that reach dramatic levels for small sample sizes.

The ARLBSL results obtained in the study demonstrate that when the process fraction of nonconforming is less than 0.2, a Standard p-Chart will be ARL-biased unless extremely high sample sizes are used, something that in real situations is not practical or simply unrealisable. 


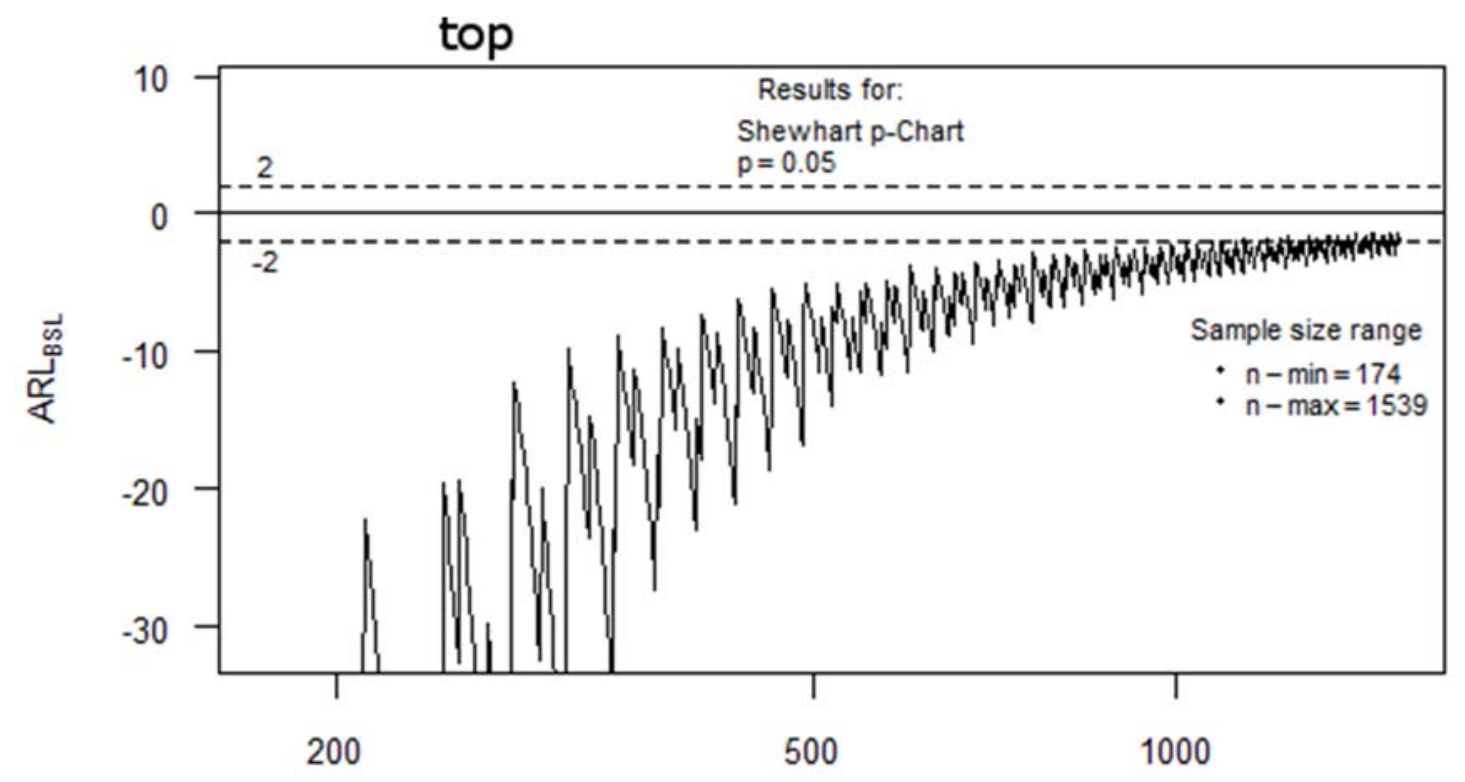

Sample size (n)

Figure 10: $\mathrm{p}$-Chart's typical ARL $\mathrm{BSL}_{\mathrm{BL}}$ behaviour for fixed $p$ and variable sample size

Figure 11 shows an example of the relation beetween the IC alpha tails and the ARLBSL for Standard p-Charts. The figure contains the $\mathrm{R}_{\alpha}$ values and their corresponding ARL BSL for $\mathrm{p}=0.05$ between a sample size range of 174 and 684 . What this figure demostrates is that the lower the difference between the IC alpha tails, the closer the ARLBSL will be to zero. This led us to conclude that to obtain quasi ARL-unbiased p-Charts the $\mathrm{R}_{\alpha}$ must be as close to one as possible.

In the following section we present a method that we have called "Kmod" through which quasi ARL-unbiased p-Charts can be obtained.

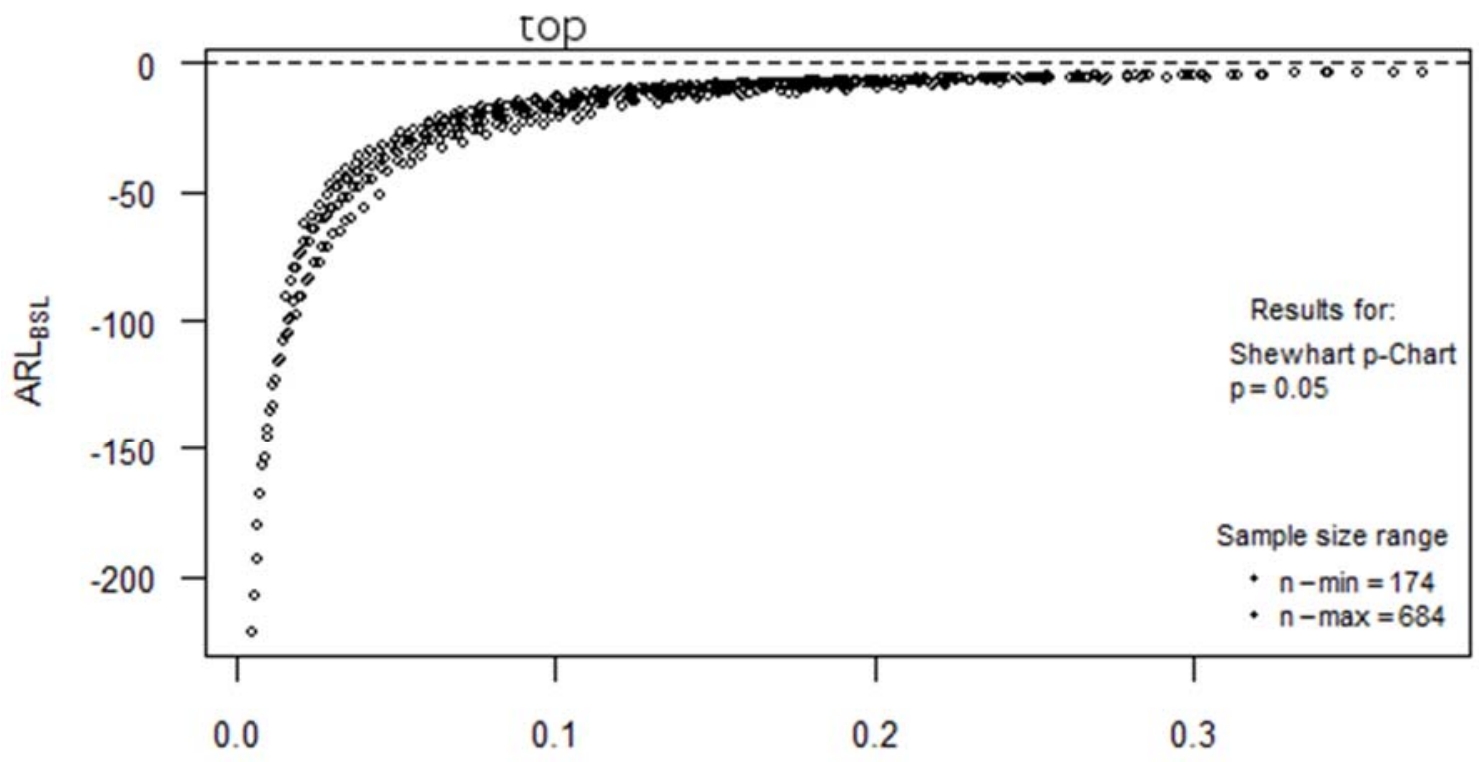

IC Alpha Tails Ratio - $R_{\alpha}$

Figure 11: Example of the effect of $\mathrm{R}_{\alpha}$ on the $A R L_{B S L}$ 


\section{Results}

\subsection{The "Kmod" method for obtaining quasi ARL-unbiased p-Charts}

\subsubsection{An adaptive $\mathrm{K}$ factor for obtaining improved control limits}

In the previous sections we explained the reasons why the control limits provided by (1) gave IC alpha tails that are far from the expected 0.00135 value, and also showed that the alpha tails ratio, $\mathrm{R}_{\alpha}$, has a direct influence on the $\mathrm{p}$-Chart's ARL-bias severity. In order to obtain control limits that would provide improved IC alpha tails values, we modified the parameter $\mathrm{K}$ in equation (1) and after extensive analysis found that very good results were obtained with control limits computed using equation (12). Charts constructed with this equation are to be known as "Kmod p-Charts". In (12) the factor $\operatorname{Kmod}_{U C L / L C L}$ is an adaptive variable whose value will depend on the binomial parameters $n$ and $p$.

Figure 12 shows an example of IC alpha tails obtained with (12) for $p=0.05$. The sample size range is the same as the one used for Figure 4 so that a comparison can be done. As can be seen the Kmod limits provide IC alpha tails that oscillate around the 0.00135 value.

Kmod Control Limits $=p \pm \operatorname{Kmod}_{U C L / L C L} \sqrt{\frac{p(1-p)}{n}}$

Where:

For upper control limit (UCL) use: $\rightarrow \operatorname{Kmod}_{U C L}=3+\frac{1}{\sqrt{n p(1-p)}}$

For lower control limit (LCL) use: $\rightarrow \operatorname{Kmod}_{L C L}=3-\frac{1.6}{\sqrt{n p(1-p)}}$

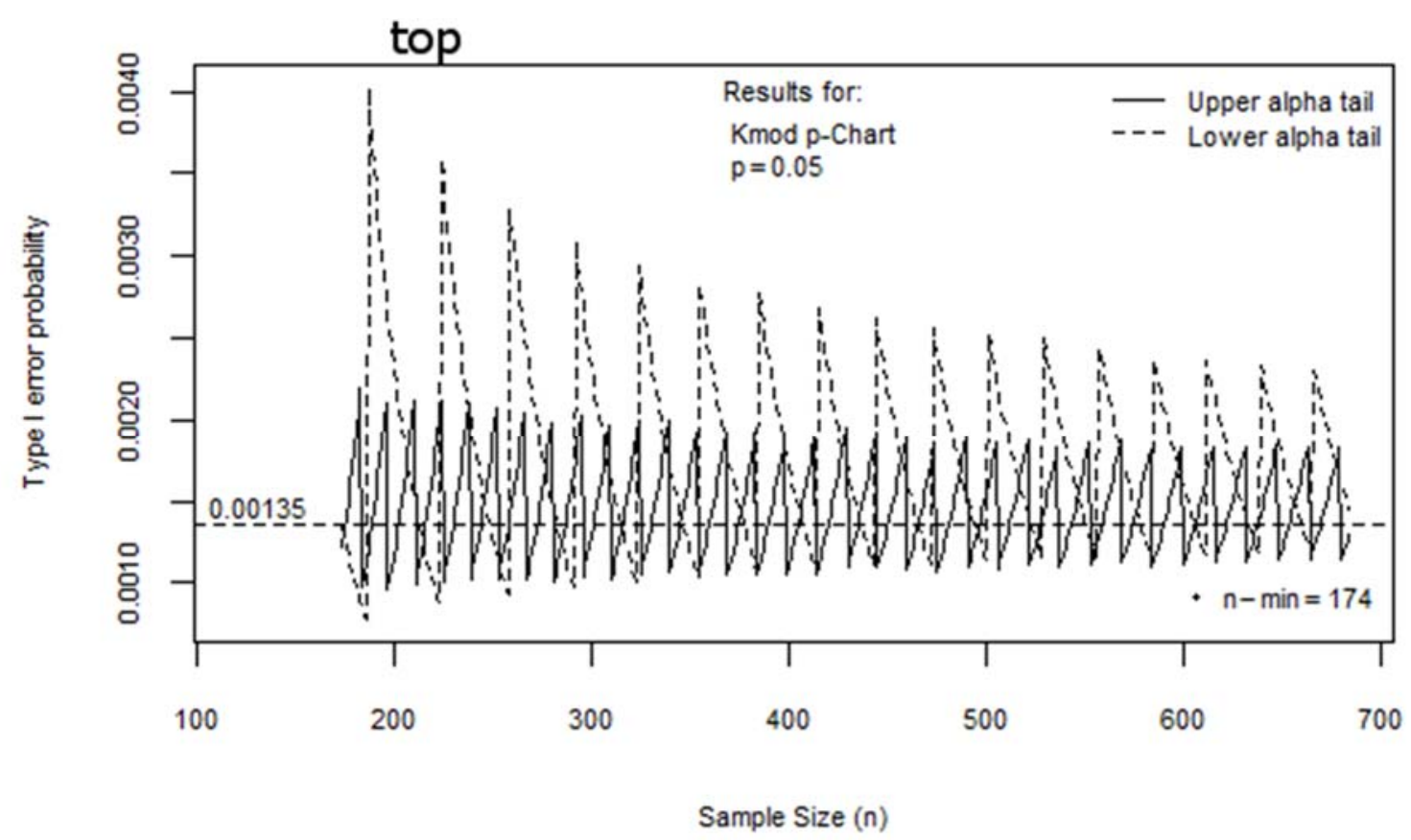

Figure 12: Example of Kmod p-Chart's typical IC alpha tails behaviour for fixed $p$ and variable $n$ 
To illustrate how the control limits computed with (12) provide improved IC alpha tails values we use the examples shown in Figures 2 and 3. For $n=244$, multiplying $n$ by the Kmod limits we obtain $\mathrm{nLCL}=3.59$ and $\mathrm{nUCL}=23.41$, observe that their corresponding discrete alpha tails values are the ones closest to 0.00135 obtaining an $\mathrm{R}_{\alpha}=1.21$. For $\mathrm{n}=245$ we obtain an $\mathrm{nLCL}=3.62$ and $\mathrm{nUCL}=23.48$, their corresponding alpha values are also the ones closest to 0.00135 providing an $\mathrm{R}_{\alpha}=1.1$. The $\mathrm{R}_{\alpha}$ values show that the disparity between the IC alpha tails has been greatly reduced and that since $R_{\alpha}=\alpha_{L} / \alpha_{U}$ the $\alpha_{L}$ is slightly higher than $\alpha_{U}$ in both cases. Figure 13 shows the ARL curves obtanied with the Kmod limits including their corresponding ARL-bias parameters, both curves are quasi ARL-unbiased with a very low ARLBSL and acceptable ARLo. Comparing these curves with the ones of Figure 7 it is obvious that a dramatic improvemement has been obtained.

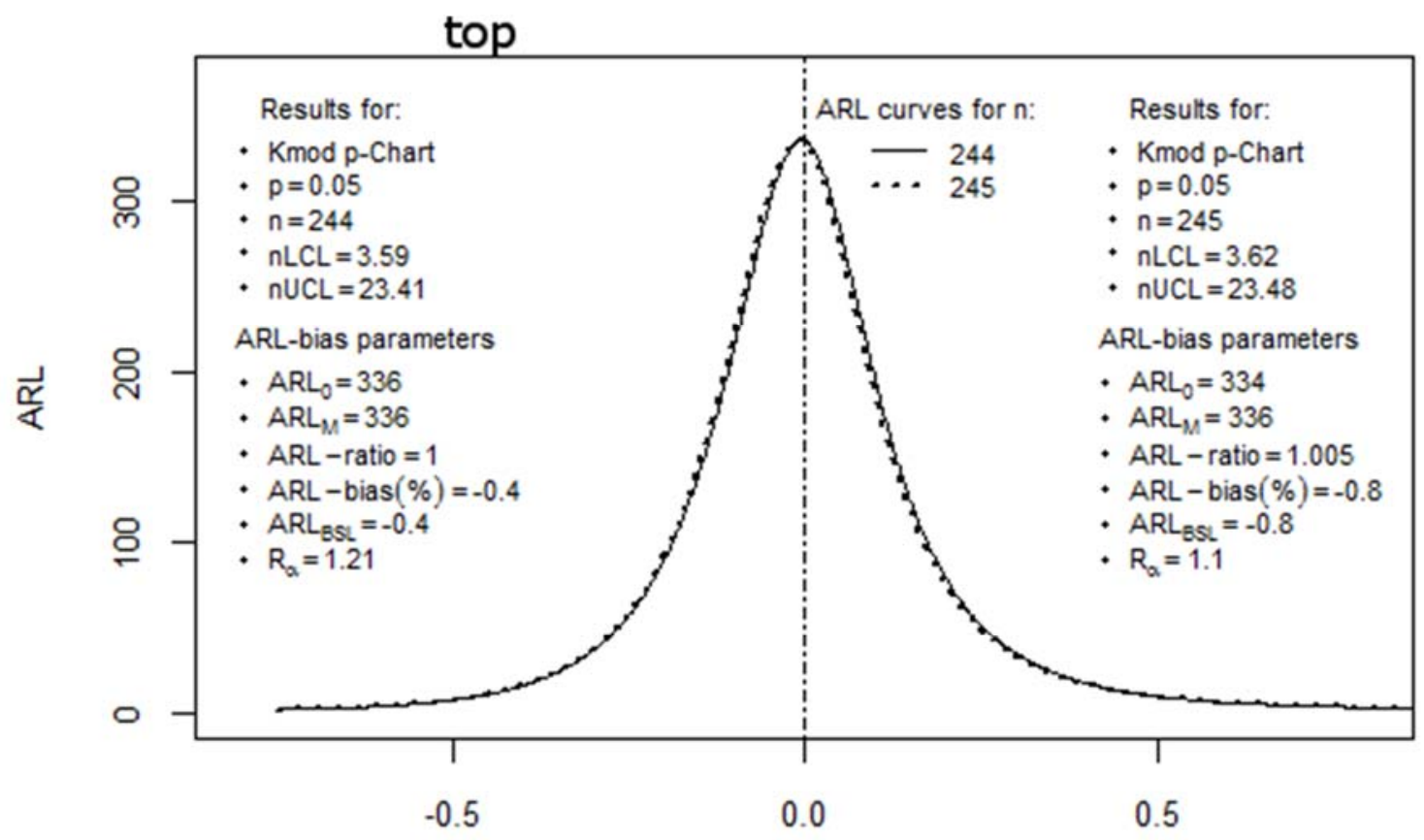

Fraction of nonconforming percentual shift $=\left(p_{1} / p\right)-1$

Figure 13: Example of quasi ARL-unbiased Kmod p-Charts

\subsubsection{Kmod p-Chart ARL ${ }_{B S L}$ and $A R L_{0}$ performance}

In order to determine the Kmod p-Chart's ARLBSL and ARLo performance for different combinations of $p$ and $n$, we carried out an extensive study computing all the relevant parameters for fractions of nonconforming between $0.005 \leq p \leq 0.20$, and for sample sizes ranges determined as follows:

1. The minimum sample size was found using equation (13). This equation was obtained by means of regression analysis and it computes, for a given $p$, the approximate minimum sample size above which a Kmod p-Chart will have a lower control limit (or LCL).

$\operatorname{Kmod} \min n=a+b * \log _{e}(p)+c * \log _{e}(q)+d * \log _{e}(p) * \log _{e}(q)$

Where: $p=$ process fraction of nonconforming; $q=1-p$

- For $0.15<\mathrm{p} \leq 0.20$ : use a minimum sample size of 25 
- For $0.017 \leq \mathrm{p} \leq 0.15: \mathrm{a}=-1630 ; \mathrm{b}=-417 ; \mathrm{c}=1593 ; \mathrm{d}=3646$

- For $0.005 \leq \mathrm{p} \leq 0.016$ : $\mathrm{a}=-15282 ; \mathrm{b}=-2636 ; \mathrm{c}=353387 ; \mathrm{d}=155947$

2. The maximum sample size was determined using (7) with $d=p / 3$.

Typical examples of the results are presented in Figures 14, 15 and 16. Figure 14 shows the ARLBSL obtained for $p=0.05$, comparing this figure with Figure 10 one can truly appreciate just how much better the ARLBSL of the Kmod p-Charts are in comparison of the Standard pCharts. Notice that $91 \%$ of the sample sizes fall within the ARLBSL criterion for "quasi ARLunbiased" and that the sample sizes that exceed this criterion are concentrated mainly in the mid-lower end. Notice also that the minimum $n$ above which the Kmod p-Chart has LCLs is 97; this is significantly lower than the 174 obtained for a Standard p-Chart. This serves to demonstrate the other advantage that the Kmod has over the Standard, which is to provide LCLs with lower sample sizes.

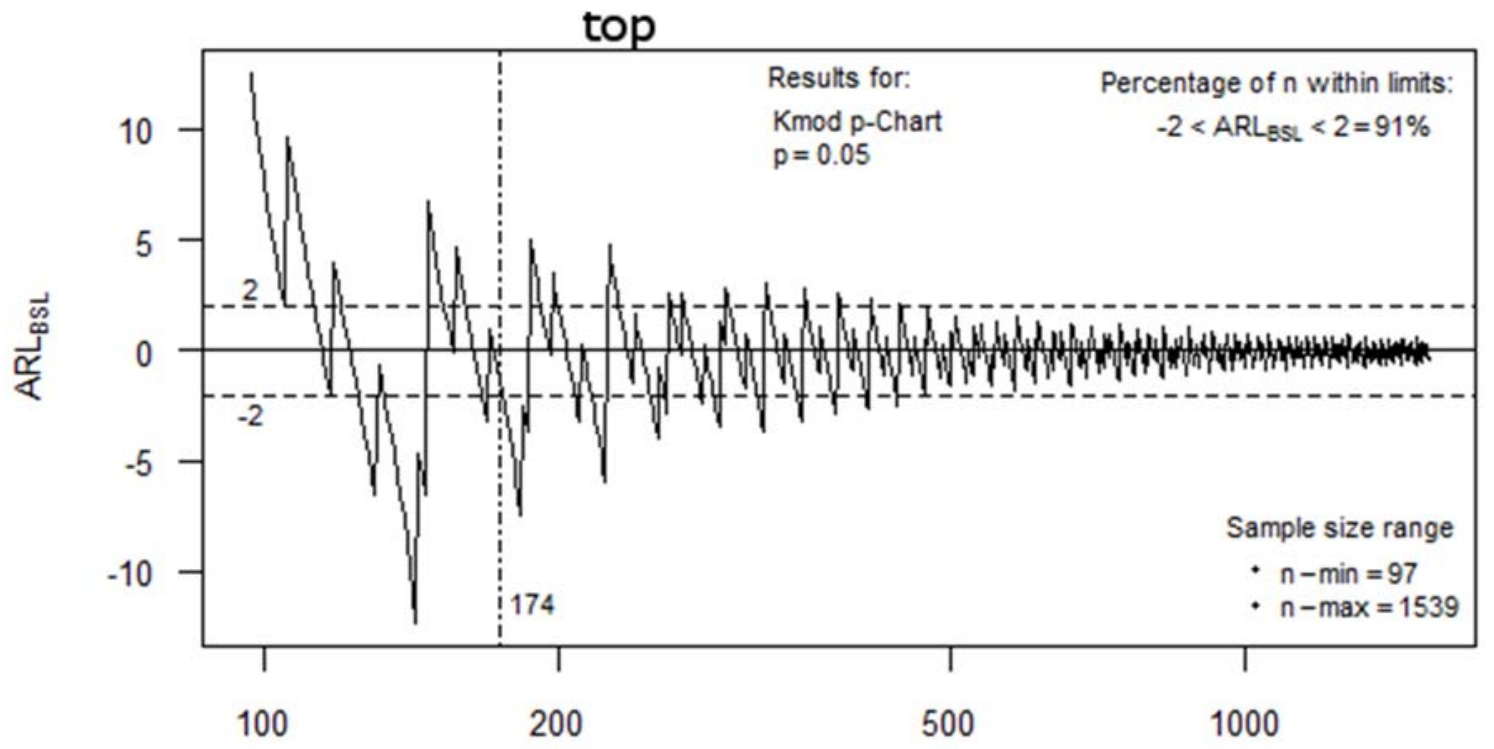

Sample size (n)

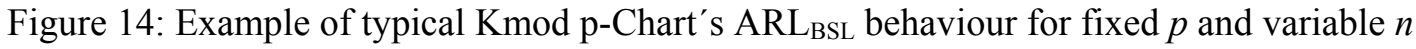

Figure 15 shows typical ARLBSL vs. $\mathrm{R}_{\alpha}$ behaviour, we include this figure to illustrate a very simple method that could be used to verify if a Kmod p-Chart has achieved the quasi ARLunbiased criteria. After extensive results analysis we found that when the IC alpha tails ratio, obtained with the Kmod limits, falls between $0.7<\mathrm{R}_{\alpha}<2.2$ the criteria $-2<$ ARL $\mathrm{BSL}<2$ is very likely to have been achieved. This $\mathrm{R}_{\alpha}$ criterion applies for all $p$ and $n$ combinations used in this study. However the following considerations must be taken into account when using this method:

a) Between $0.7<\mathrm{R}_{\alpha}<1$ a low proportion of sample sizes would exceed the criteria $-2<$ ARLBSL, reaching maximum levels around ARLBSL $\approx-3$. However, we consider that even at these levels the ARL curves would have acceptable ARL-bias(\%) and ARLratios.

b) When $R_{\alpha}$ is close to around 0.7 or 2.2 , care must be exercised because at those extremes some $p$ and $n$ combinations could: i) Provide an ARLBSL value that excessively exceeds

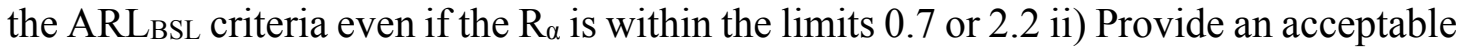
ARLBSL value even if the $\mathrm{R}_{\alpha}$ is outside the limits 0.7 or 2.2 . We would recommend that 
in these cases the actual ARLBSL be computed or alternatively the method of changing the sample size, described below, be applied to obtain better $\mathrm{R}_{\alpha}$.

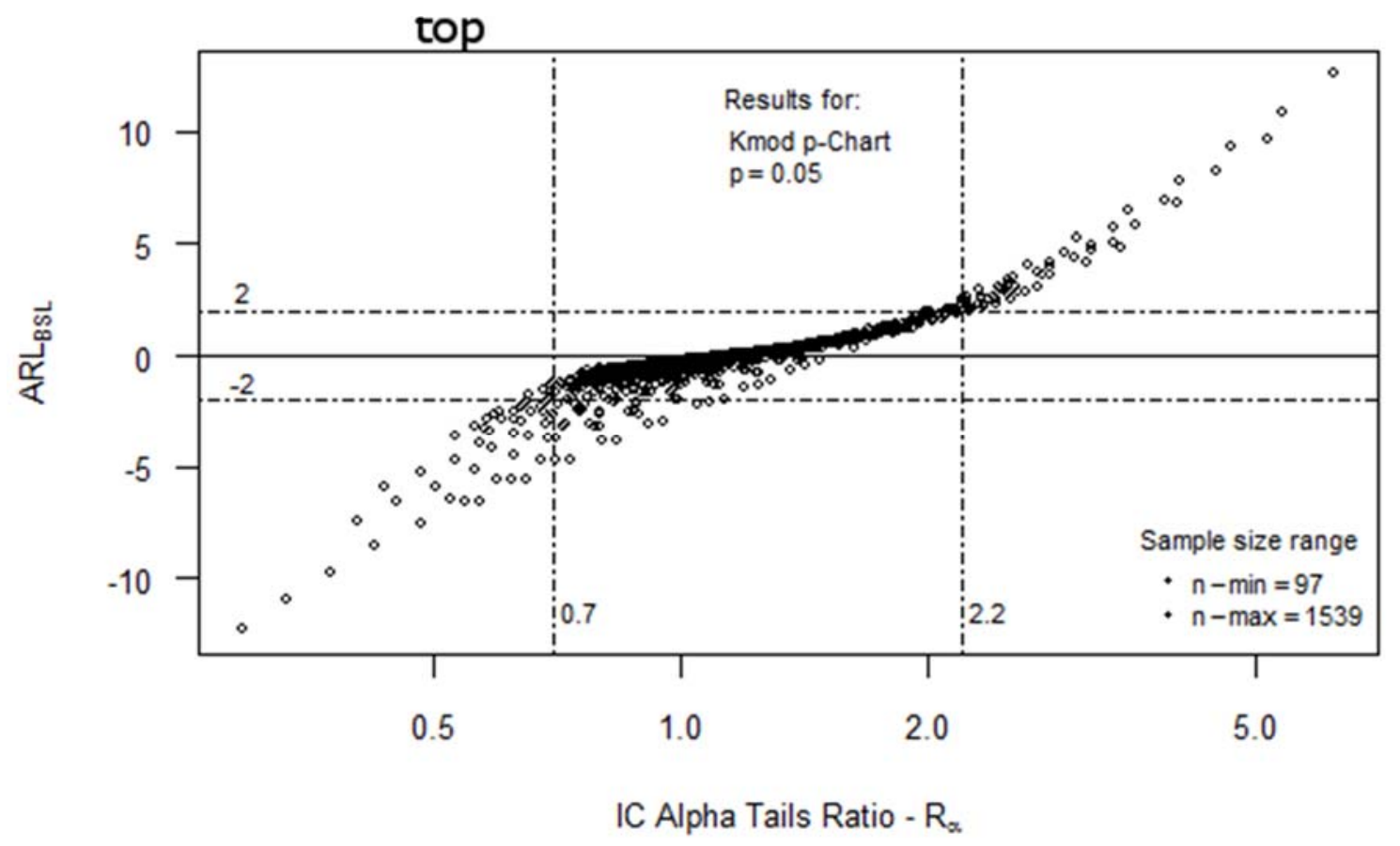

Figure 15: Example of typical behaviour of $A R L_{B S L}$ vs. $R_{\alpha}$ in Kmod p-Charts

Figure 16 shows typical ARLo vs. $\mathrm{R}_{\alpha}$ behaviour, we include this figure to show the Kmod pChart's ARL 0 performance. Notice that $93 \%$ of the samples sizes used for $p=0.05$ are within $250<\mathrm{ARL}_{0}<450$, which is an $\mathrm{ARL}_{0}$ range we considered to be acceptable for process monitoring. However, the figure also demonstrates that for some $p$ and $n$ combinations, the ARLo maybe too low or too high even if the Kmod p-Chart is quasi ARL-unbiased, for this reason we strongly recommend to always compute the ARL 0 . If the ARL 0 is not satisfactory then we would recommend applying the "varying sample size method", as described below, until a satisfactory value is obtained. 


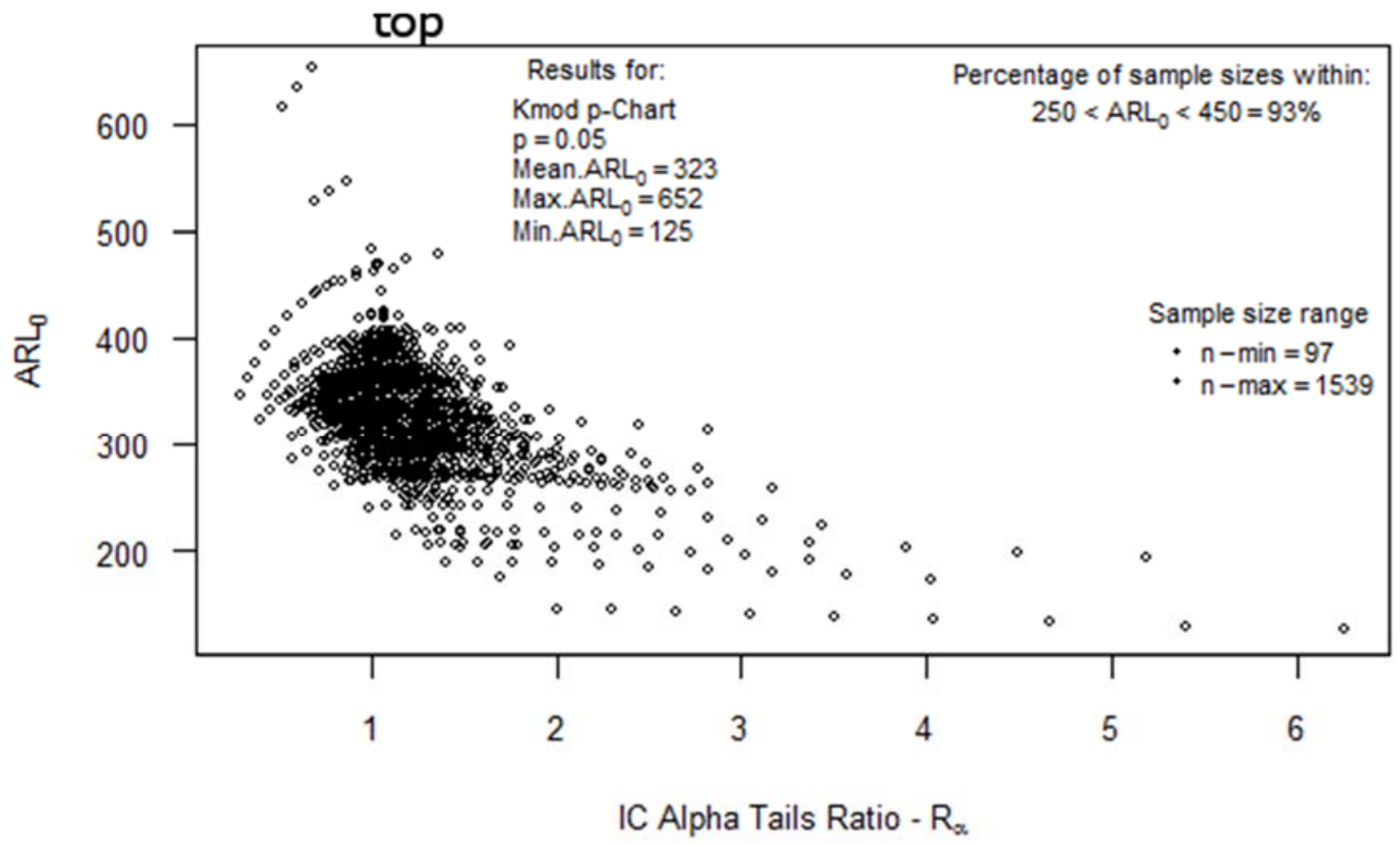

Figure 16: Example of typical behaviour of $\mathrm{ARL}_{0}$ vs. $\mathrm{R}_{\alpha}$ in Kmod p-Charts

Table 1 contains a summary of the results obtained in the study carried out to establish the Kmod p-Chart ARLBSL and ARLo performance. As can be seen excellent results are obtained between $0.005 \leq \mathrm{p} \leq 0.10$, a $p$ range commonly found in modern production processes. The performance gradually reduces for $\mathrm{p}>0.10$, for this reason we would not recommend using the Kmod $\mathrm{p}$-Chart above $\mathrm{p}=0.20$.

When an $n$ and $p$ combination does not provide adequate Kmod p-Charts, then the method of "modifying the sample size" can be applied. This method is explained in the next section. 
Table 1: Summary of Kmod p-Charts $A R L_{B S L}$ and $A_{R L}$ performance

\subsubsection{Obtaining quasi ARL-unbiased Kmod p-Charts by modifying the sample size}

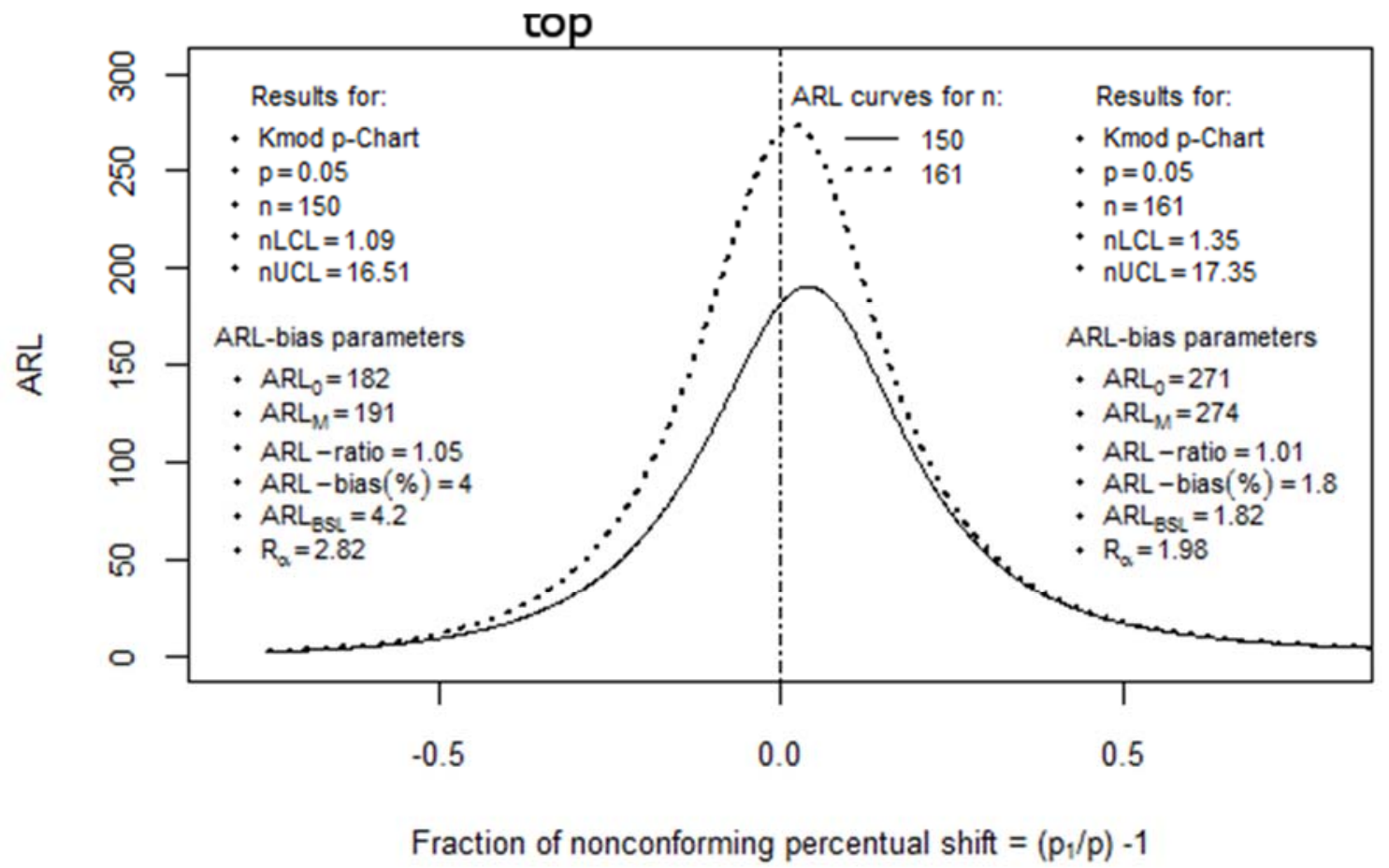

Figure 17: Example of how to obtain Kmod p-Charts by modifying the sample size

Figure 17 shows an example of how quasi ARL-unbiased Kmod p-Charts can be obtained by varying the sample size. Let's suppose we wish to use a Kmod p-Chart to monitor a process where $p=0.05$ and that we would like to use an $n=150$. The first step would be to calculated the IC alpha tails, with these in hand it would be revealed that $\mathrm{R}_{\alpha}=2.82$, which exceeds the $0.7<$ $\mathrm{R}_{\alpha}<2.2$ criteria for quasi ARL-unbiased, and that the $\mathrm{ARL}_{0}$ is 182 ; which is a far too low ARL $_{0}$. These two results should be sufficient to determine that satisfactory process monitoring cannot be achieved with this $p$ and $n$ combination.

To illustrate the method of varying the sample size we refer to Figure 14, in it one could locate the $\mathrm{n}=150$ and identify that it's corresponding ARLBSL $\approx 4.2$. Now notice that the ARLBSL for several $n$ between 150 and 174 fall within the \pm 2 criteria, we searched within that range for an $n$ closest to 150 that would provide optimum $\mathrm{R}_{\alpha}$ and $\mathrm{ARL}_{0}$ values and found that $\mathrm{n}=161$ gave the best results. This is the sample size used for the second ARL curve plotted in Figure 17, notice that the $\mathrm{R}_{\alpha}=1.98$, which is within the $0.7<\mathrm{R}_{\alpha}<2.2$ criteria and that the ARLo is 271 . As can be seen with $n=161$ a "quasi ARL-unbiased" Kmod p-Chart with an acceptable ARLo has been obtained. So, to summarize; if a $p$ and $n$ combination provides $\mathrm{R}_{\alpha}$ and/or ARL 0 that are likely to produced inadequate Kmod p-Charts then simply vary the sample size until satisfactory values are obtained.

\subsection{Comparison of alternative $p$-Charts $A R L_{B S L}$ and $A R L_{0}$ performance}

One of the main objectives of our research was to identify the most suitable alternative p-Chart out of the ones mentioned in the introduction section. To that list of alternative charts we added the Kmod p-Chart. The performance comparison was made on basis of their ARLBSL and ARLo. Hence, these parameters were computed for each alternative chart for nonconforming fractions 
between $0.005 \leq p \leq 0.10$ and for sample size ranges specific for each $p$, with minimum and maximum sample sizes determined as follows:

- Minimum $n$ : Obtained with $\mathrm{d}=0.99 \mathrm{p}$ in equation (7).

Since it is a comparative study we concluded that the minimum sample size had to guaranteed the existence of a LCL for all the alternative charts, $d=0.99 p$ in equation ( 7 ) ensures this condition and provides an adequate lower sample size.

- Maximum $n$ : Obtained with $\mathrm{d}=\mathrm{p} / 2$ in equation (7).

Based on the fact that it is with lower sample sizes that the charts performed the worst, we concluded that the upper sample size should not be too high to allow a fare comparison between them. We estimated that $d=p / 2$ in (7) provides a sufficiently high sample size.

For reason of conciseness, we include only the results for the charts that performed best. Prior to presenting the results we show typical IC alpha tail behaviour for each of them.

\subsubsection{The Regression-Based p-Chart}

Ryan and Schwertmann [1] utilised regression methods to develop equation (14) whereby improved, three sigma, IC alpha tails could be obtained.

$$
R B \text {-Chart Control Limits }=\frac{1}{n}[a+b(n p)+c \sqrt{n p}]
$$

Where:

- For upper control limit $\rightarrow a=0.6195 ; b=1.00523 ; c=2.983$

- For lower control limit $\rightarrow a=2.9529 ; b=1.01956 ; c=3.2729$

- Chart's Mid line $\rightarrow p=$ process fraction of nonconforming

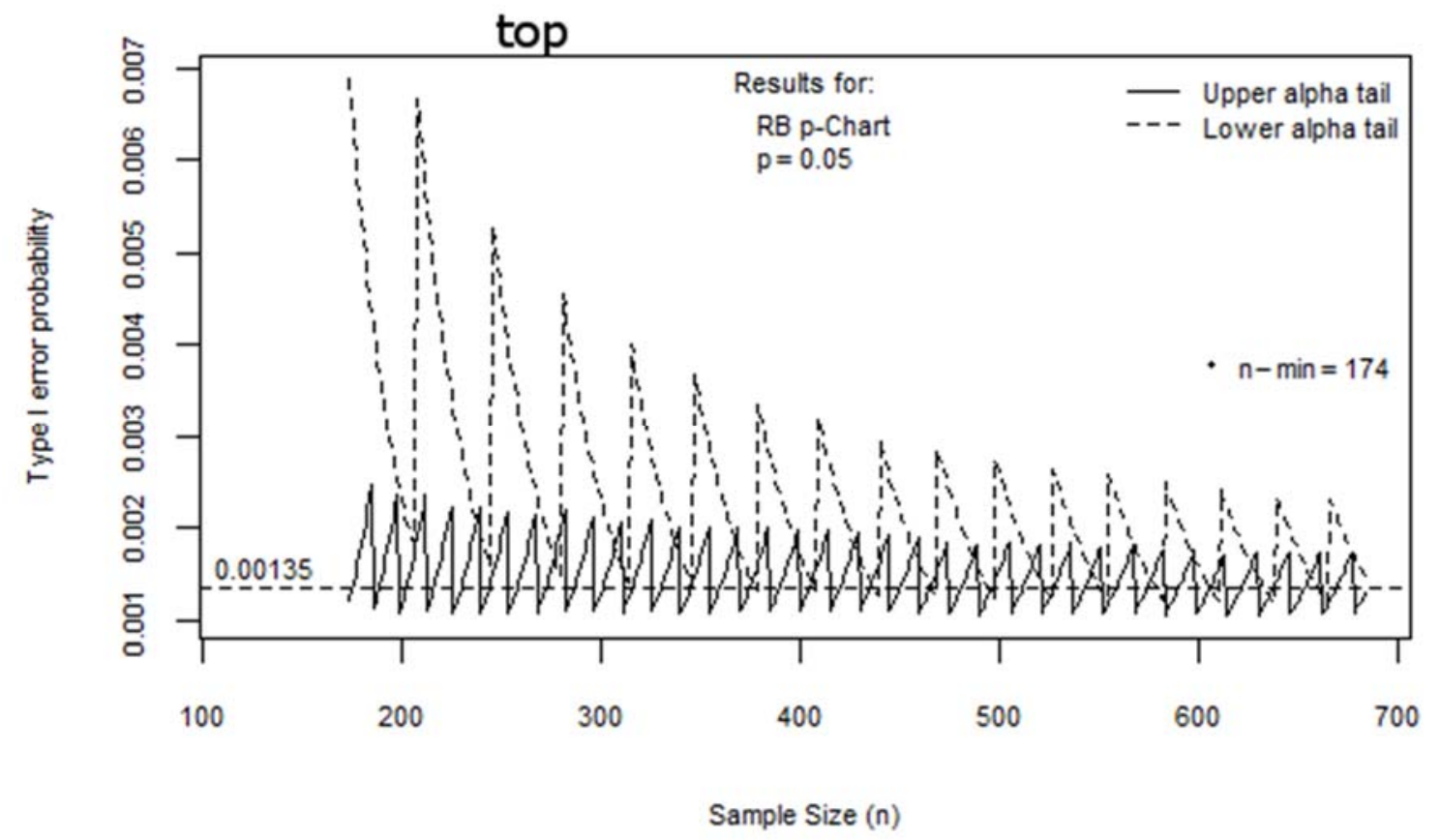

Figure 18: Example of RB p-Chart's typical IC alpha tails behaviour for fixed $p$ and variable $n$

Figure 18 shows an example of typical IC alpha tails obtained by means of (14). Comparing these tails with the ones of Figure 4 one can see that the upper alpha tail now oscillates around 
0.00135 whilst the lower tail oscillates mainly above it. Notice that in many instances both tails would have values above 0.00135 and that at lower sample sizes the lower tail peak values surpass those of the Standard chart. Let's recall that $A R L_{0}=1 /\left(\alpha_{L}+\alpha_{U}\right)$, hence if the $\alpha_{L}+$ $\alpha_{U}$ value is excessively above 0.0027 then the result would be an ARLo much below the nominal 370.

\subsubsection{The Corner-Fisher expansion p-Chart}

Winterbottom [6] made use of the Corner-Fisher expansion to develop equation (13) by which control limits that provide improved IC alpha tails could be obtained.

$$
\text { CF } p \text {-Chart Control Limits }=p \pm K \sqrt{\frac{p(1-p)}{n}}+\frac{4(1-2 p)}{3 n}
$$

Where:

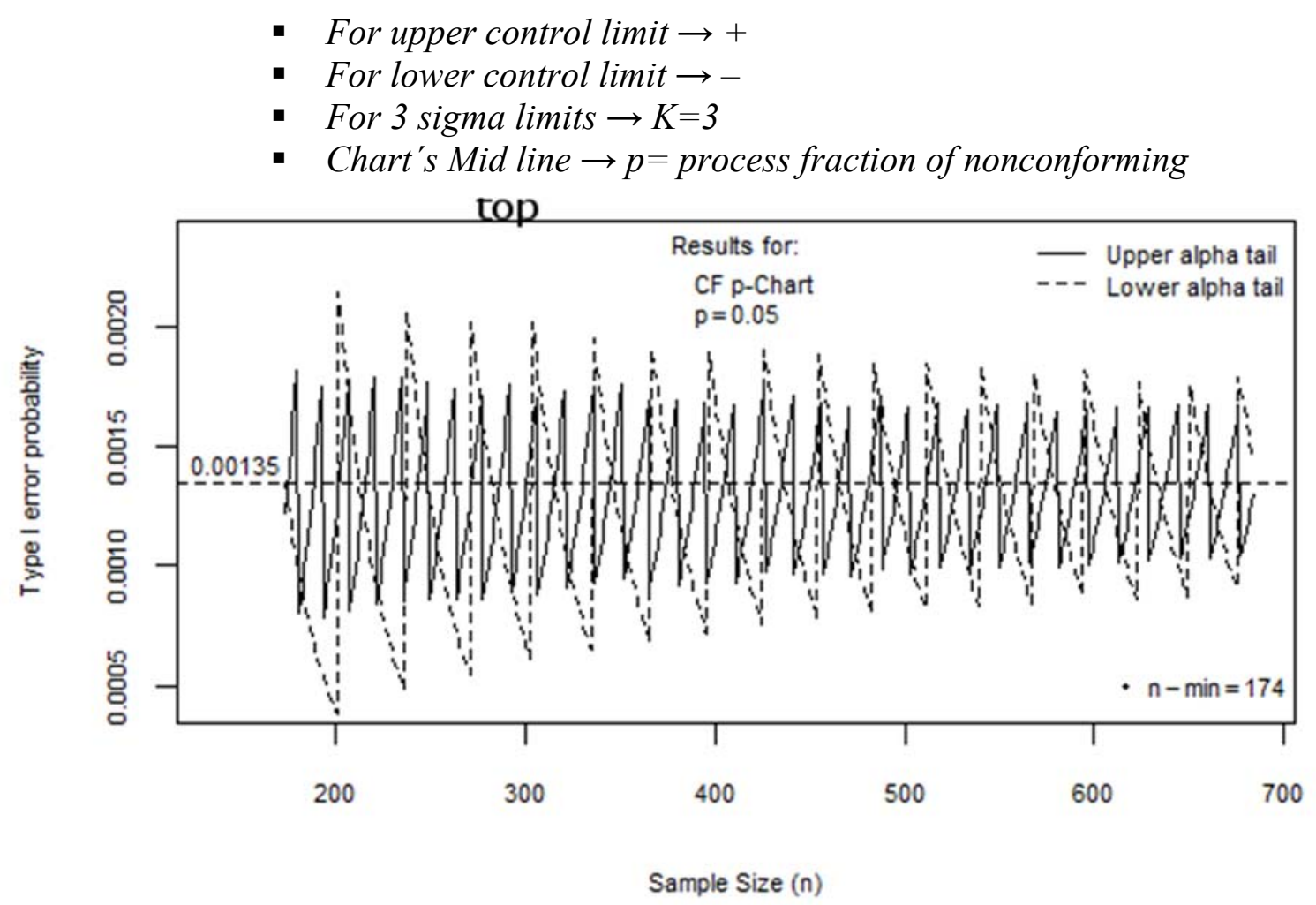

Figure 19: Example of CF p-Chart's typical IC alpha tails behaviour for fixed $p$ and variable $n$

Figure 19 shows an example of typical IC alpha tails obtained by means of (13). Comparing these tails with the ones of Figure 4 one can see that now both tails oscillate around 0.00135 . Notice that in many instances both tails would have values far below 0.00135 . Thus, since $A R L_{0}=1 /\left(\alpha_{L}+\alpha_{U}\right)$, then a $\alpha_{L}+\alpha_{U}$ value excessively below 0.0027 will result in an ARL 0 significantly higher than the nominal 370 .

\subsubsection{The Arcsine transformation equivalent p-Chart}

A strategy often used to make non-nomal data resemble normal data is to use a transformation. For binomial data a transformation that makes it approach normality is the Arcsine. The control limits for this chart are computed by means of equation (15), [2, p. 188\&189] 
Arcsine Chart Control Limits $=\operatorname{Sin}^{-1}(\sqrt{p}) \pm \frac{3}{2 \sqrt{n}}$

Where:

- For upper control limit $\rightarrow+$

- For lower control limit $\rightarrow-$

- Chart's Mid line $=\operatorname{Sin}^{-1}(\sqrt{p})$

- $y=\operatorname{Sin}^{-1} \sqrt{\frac{x}{n}} \rightarrow$ Variable plotted on the Arcsine Chart

- $\mathrm{x}=$ number of nonconforming; $\mathrm{n}=$ sample size; $\mathrm{p}=$ process fraction of nonconforming.

A method for determining the Arcsine chart's performance is to find its equivalent conventional p-Chart's limits, which can be done through equation (16), and then used these to obtain the IC alpha tails as we've done for the conventional p-Chart, [13, p. 512].

Arcsine equivalent conventional $p$-Chart limits $=\operatorname{Sin}^{2}\left[\operatorname{Sin}^{-1}(\sqrt{p}) \pm \frac{3}{2 \sqrt{n}}\right]$

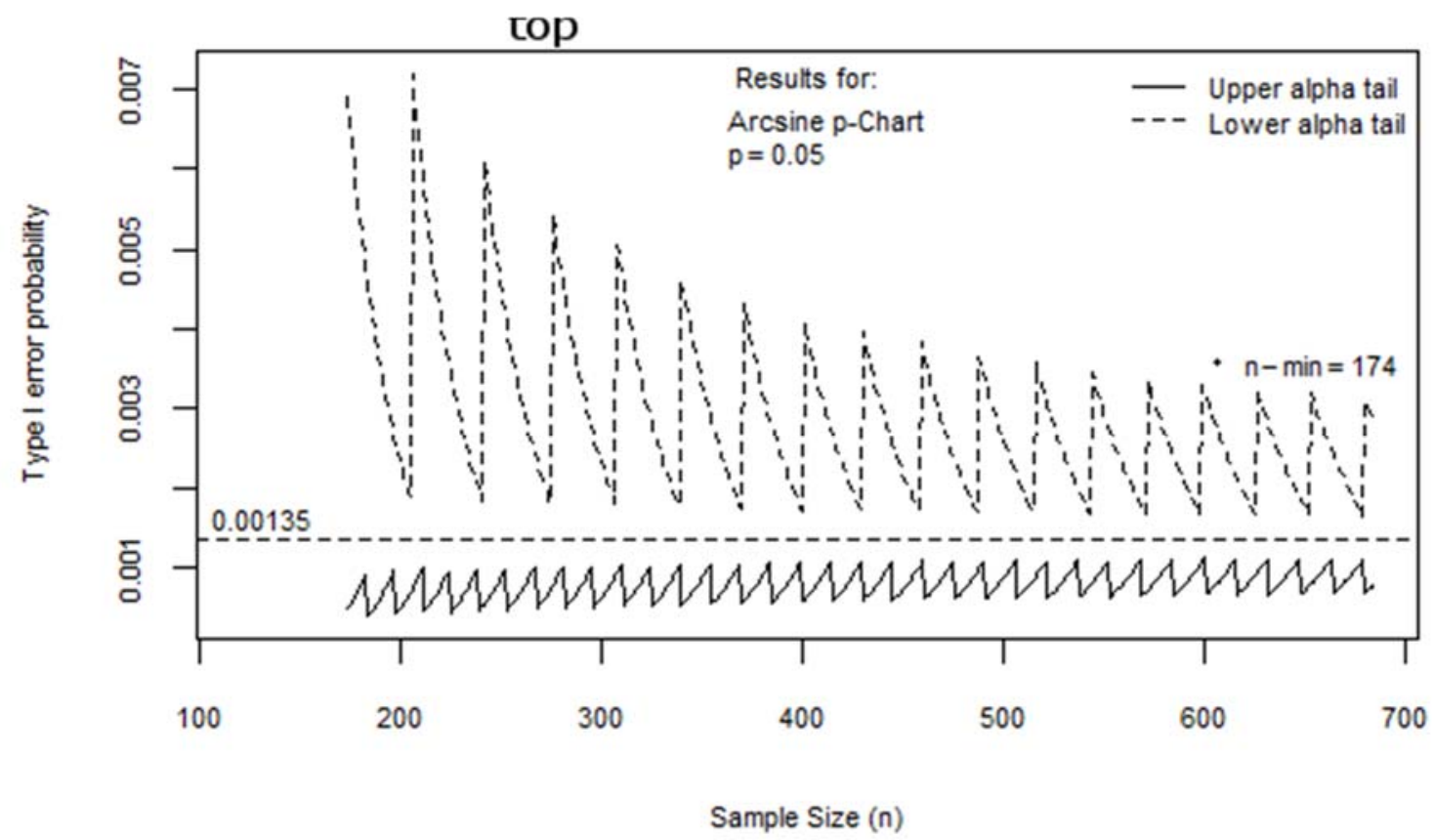

Figure 20: Example of Arcsine p-Chart's typical IC alpha tails behaviour for fixed $p$ and variable $n$

Figure 20 shows an example of typical IC alpha tails obtained by means of (15). Comparing these tails with those of Figure 4 one can see that it is the lower tail that now fluctuates above 0.00135 and that at low sample sizes this tail has upper peak values that surpass those of the Standard chart. On the other hand the upper tail now fluctuates just below 0.00135. In general the alternative charts based on transformations performed poorly in terms of the ARL BSL. The Arcsine results are included only for comparison completeness.

\subsubsection{A summary of the $A R L_{B S L}$ and $A R L_{0}$ performance comparison results}

Table 2 summarises the results obtained in our ARLBSL and ARL 0 comparative study of the Kmod, CF, RB and Arcsine p-Charts. To aid interpret the results, lets concentrate in the values for $\mathrm{p}=0.01$, as can be seen in the sample size range $909 \leq n \leq 3564$ the proportion of charts that 
would be "quasi ARL-unbiased" is $82 \%$ for the Kmod, $88 \%$ for the RB, $75 \%$ for the CF and $11 \%$ for the Arcsine. Thus, based solely on the ARLBSL it would appear that the RB chart provides better results than the other three. However, closer inspection of the ARLo performance reveals that only $45 \%$ of those "quasi ARL-unbiased" RB charts would have an $\mathrm{ARL}_{0}$ between $250<\mathrm{ARL}_{0}<450$, in contrast to the $87 \%$ for the Kmod. Taking into account the ARLBSL and the ARLoperformances it is clear that the Kmod is superior to all the others.

Even more useful comparative information can be extracted from the ARLo quartiles. For example, for $\mathrm{p}=0.01$, the quartiles for the RB method show that up to $50 \%$ of the charts that meet the "quasi ARL-unbiased" criteria would have ARLo values below 242 and that up to 25\% of them would be below 222. The reason for these low ARL 0 was explained in the analysis of the IC alpha tails obtained with the RB method, see Figure 18.

Analysing the results for all $p$ values and taking into account the ARLBSL and the ARLo performances; it can be easily deduced that the Kmod method provides "quasi ARL-unbiased" charts with adequate ARL 0 more often than any of the other three. To this fact it must be added that the Kmod offers a simple and effective way of verifying if a chart has achieved the quasi ARL-unbiased criteria and that in case it hadn't, it also offers the method of varying the sample size.

At this point we should mention that Morais [15] [16] proposed a method based on binomial quantile limits and randomized probabilities, which could be used to obtain ARL-unbiased pCharts (charts with ARL ${ }_{B S L}=0$ ). Although this method doesn't fall within this paper's research criteria of $\mathrm{p}$-Charts whose limits are computed thorough closed-form equations and data transformation methods, nevertheless we believe it's worth mentioning it.

Table 2: Summary of $\mathrm{ARL}_{\mathrm{BSL}}$ and $\mathrm{ARL}_{0}$ performance results for various alternative $\mathrm{p}$-Charts

\section{Conclusions}

The main objective of the research project summarised in this paper was to find, in accordance to the criteria set out in the introduction section, the most idoneous alternative to the Standard p-Chart. To this end we firstly we identified suitable existing alternatives and then developed: i) The Average Run Length bias severity level (ARLBSL) ii) An ARLbsL criteria for establishing a p-Chart's "quasi ARL-unbiased" condition, and iii) The Kmod p-Chart.

ARLBSL performance results for the Standard p-Chart were extremely poor. Thus, it is our opinion that this chart should be avoided if monitoring process deterioration and improvement is required. Out of the existing alternative charts listed in the introduction, it was found that the one based on the Corner-Fisher expansion performed best in terms of both ARLBSL and ARL 0 ; however, even this chart falls short of the "quasi ARL-unbiased" criteria, especially at low sample sizes. Hence, if this chart is used without any previous ARL analysis; unwittingly, one could be monitoring a process with an ARL-biased Chart.

The new "Kmod p-Chart" performed best in terms of ARLBSL and ARLo compared to all other listed alternative charts. However, in certain instances, especially when low sample sizes are used, this chart could be moderately ARL-biased. In order to verify its ARL-bias condition, a very simple method based on its IC alpha tail ratio was developed; this method enables a practitioner to establish a chart's monitoring suitability without the need of a full ARL analysis. 
In addition, for cases where the Kmod p-Chart is ARL-biased, a method for correcting this condition, based on varying the sample size, was also developed.

\section{References}

[1] Ryan TP, Schwertman NC. Optimal limits for attribute control charts. Journal of Quality Technology 1997; 29:86-98.

[2] Ryan TP. Statistical Methods for Quality Improvement 3th ed. Hoboken, New Jersey: John Wiley \& Sons, 2011.

[3] Montgomery DC. Introduction to Statistical Quality Control 6th ed. Hoboken, New Jersey: Jhon Wiley \& Sons, 2009.

[4] Schader M, Schmid F. Two rules of thumb for the approximation of the binomial. The American Statistician 1989; 43:23-24.

[5] Ryan TP. Statistical Methods for Quality Improvement, New York: Jhon Wiley \& Sons, 1989.

[6] Winterbottom A. Simple Adjustments To Improve Control Limits On Attribute Charts. Quality and Reliability Engineering International 1993; 9:105-109.

[7] Chen G. An improved chart through simple adjustments. Journal of Quality Technology 1998; 30:142-151.

[8] Park C. An Improved p Chart Based on the Wilson Interval. Journal of Statistics and Management Systems 2013, 16: 201-221.

[9] C. P. Quesenberry, "SPC Q Charts for a Binomial Parameter: Short and Long Runs," Journal of Quality Technology, vol. 23, pp. 239-246, 1991.

[10] Quesenberry CP.On properties of binomial Q charts for attributes. Journal of Quality Technology 1995; 27: 204-213.

[11] Mitra A. Fundamentals of Quality Control and Improvement 3th ed. Hoboken, NJ: Jhon Wiley \& Sons, 2008.

[12] Duncan AJ. Quality Control and industrial Statistics 5th ed. Homewood: Irwin, 1986.

[13] Acosta-Mejía C. Improved p-charts to monitor process quality. IIE Transactions 1999 31: 509-516.

[14] Qiu P. Introduction to Statistical Process Control. Boca Raton, FL: CRC Press, 2014.

[15] Morais MC. An ARL-Unbiased np-Chart. Economic Quality Control 2016; 31:11-21. 
[16] Morais MC. ARL-unbiased geometric and CCCG control charts. Sequential Analysis 2017; 36: 513-527. 
Table 3: Summary of Kmod p-Charts $\mathrm{ARL}_{\mathrm{BSL}}$ and $\mathrm{ARL}_{0}$ performance

\begin{tabular}{|c|c|c|c|c|c|c|c|}
\hline \multirow[b]{2}{*}{$p$} & \multicolumn{2}{|c|}{$\begin{array}{l}\text { Sample Size } \\
\text { range }\end{array}$} & \multirow{2}{*}{$\begin{array}{c}\% \text { of } n \text { within } \\
-2<\text { ARL }_{B S L}<2\end{array}$} & \multicolumn{4}{|c|}{$\begin{array}{c}\text { Kmod p-Chart } A R L_{0} \text { performance for all } \\
n \text { within sample size range }\end{array}$} \\
\hline & n-min & n-max & & Min & $\begin{array}{c}\text { Average } \\
\text { ARL }_{0} \\
\end{array}$ & Max & $\begin{array}{c}\% \text { of } n \text { within } \\
250<\text { ARL }_{0}<450\end{array}$ \\
\hline 0.20 & 25 & 324 & $69 \%$ & 88 & 289 & 468 & $78 \%$ \\
\hline 0.18 & 25 & 369 & $75 \%$ & 112 & 297 & 417 & $83 \%$ \\
\hline 0.15 & 26 & 459 & $81 \%$ & 65 & 300 & 544 & $82 \%$ \\
\hline 0.12 & 39 & 594 & $87 \%$ & 120 & 310 & 488 & $88 \%$ \\
\hline 0.10 & 47 & 729 & $90 \%$ & 112 & 315 & 614 & $90 \%$ \\
\hline 0.08 & 58 & 931 & $91 \%$ & 102 & 317 & 554 & $88 \%$ \\
\hline 0.05 & 97 & 1539 & $91 \%$ & 125 & 323 & 652 & $93 \%$ \\
\hline 0.02 & 257 & 3969 & $91 \%$ & 128 & 327 & 575 & $92 \%$ \\
\hline 0.01 & 523 & 8019 & $90 \%$ & 160 & 328 & 551 & $93 \%$ \\
\hline 0.005 & 1054 & 16119 & $90 \%$ & 162 & 329 & 982 & $95 \%$ \\
\hline
\end{tabular}


Table 4: Summary of $\mathrm{ARL}_{\mathrm{BSL}}$ and $\mathrm{ARL}_{0}$ performance results for various alternative $\mathrm{p}$-Charts

\begin{tabular}{|c|c|c|c|c|c|c|c|c|c|}
\hline \multirow{3}{*}{$\begin{array}{c}\text { P } \\
\text { Chart }\end{array}$} & \multirow{3}{*}{$p$} & \multirow{3}{*}{$\begin{array}{c}\text { n range } \\
\min \\
\max \end{array}$} & \multirow{3}{*}{$\begin{array}{c}\% \text { of charts } \\
\text { within } \\
-2<\text { ARLBSL }_{\text {B }}<2\end{array}$} & \multicolumn{6}{|c|}{$\begin{array}{c}\text { ARL }_{0} \text { performance of charts that } \\
\text { fall within: }-2<\text { ARL }_{B S L}<2\end{array}$} \\
\hline & & & & \multicolumn{5}{|c|}{ 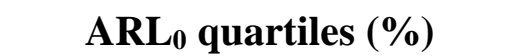 } & \multirow{2}{*}{$\begin{array}{l}\% \text { of } n \text { within } \\
250<\text { ARL } 0_{0}<450\end{array}$} \\
\hline & & & & $\mathbf{0}$ & 25 & 50 & 75 & 100 & \\
\hline Kmod & \multirow{4}{*}{0.005} & \multirow{4}{*}{$\begin{array}{l}1827 \\
7164\end{array}$} & $80 \%$ & 198 & 285 & 308 & 356 & 452 & $91 \%$ \\
\hline RB & & & $88 \%$ & 121 & 218 & 237 & 280 & 354 & $43 \%$ \\
\hline $\mathrm{CF}$ & & & $75 \%$ & 270 & 350 & 379 & 432 & 592 & $81 \%$ \\
\hline Arcsine & & & $11 \%$ & 277 & 331 & 360 & 374 & 409 & $100 \%$ \\
\hline Kmod & \multirow{4}{*}{0.01} & \multirow{4}{*}{$\begin{array}{l}909 \\
3564\end{array}$} & $82 \%$ & 173 & 286 & 306 & 353 & 463 & $87 \%$ \\
\hline RB & & & $88 \%$ & 123 & 222 & 242 & 286 & 363 & $45 \%$ \\
\hline $\mathrm{CF}$ & & & $75 \%$ & 276 & 344 & 384 & 432 & 605 & $80 \%$ \\
\hline Arcsine & & & $11 \%$ & 284 & 332 & 358 & 377 & 416 & $100 \%$ \\
\hline Kmod & \multirow{4}{*}{0.02} & \multirow{4}{*}{$\begin{array}{c}450 \\
1764\end{array}$} & $84 \%$ & 180 & 272 & 311 & 352 & 484 & $87 \%$ \\
\hline RB & & & $87 \%$ & 128 & 232 & 254 & 299 & 381 & $55 \%$ \\
\hline $\mathrm{CF}$ & & & $75 \%$ & 251 & 343 & 393 & 429 & 581 & $82 \%$ \\
\hline Arcsine & & & $11 \%$ & 277 & 327 & 349 & 371 & 412 & $100 \%$ \\
\hline Kmod & \multirow{4}{*}{0.03} & \multirow{4}{*}{$\begin{array}{c}297 \\
1164\end{array}$} & $85 \%$ & 186 & 269 & 319 & 350 & 506 & $87 \%$ \\
\hline $\mathrm{RB}$ & & & $87 \%$ & 133 & 243 & 265 & 314 & 384 & $64 \%$ \\
\hline $\mathrm{CF}$ & & & $76 \%$ & 262 & 336 & 382 & 433 & 608 & $82 \%$ \\
\hline Arcsine & & & $12 \%$ & 271 & 322 & 349 & 371 & 411 & $100 \%$ \\
\hline Kmod & \multirow{4}{*}{0.04} & \multirow{4}{*}{$\begin{array}{l}220 \\
864\end{array}$} & $83 \%$ & 195 & 272 & 315 & 346 & 480 & $93 \%$ \\
\hline $\mathrm{RB}$ & & & $85 \%$ & 139 & 255 & 278 & 331 & 404 & $79 \%$ \\
\hline $\mathrm{CF}$ & & & $75 \%$ & 266 & 344 & 381 & 444 & 601 & $80 \%$ \\
\hline Arcsine & & & $14 \%$ & 285 & 331 & 345 & 368 & 417 & $100 \%$ \\
\hline Kmod & \multirow{4}{*}{0.05} & \multirow{4}{*}{$\begin{array}{l}174 \\
684\end{array}$} & $84 \%$ & 175 & 274 & 309 & 356 & 482 & $90 \%$ \\
\hline $\mathrm{RB}$ & & & $84 \%$ & 145 & 267 & 290 & 345 & 425 & $81 \%$ \\
\hline $\mathrm{CF}$ & & & $76 \%$ & 270 & 352 & 378 & 445 & 630 & $76 \%$ \\
\hline Arcsine & & & $16 \%$ & 276 & 336 & 355 & 367 & 425 & $100 \%$ \\
\hline Kmod & \multirow{4}{*}{0.10} & \multirow{4}{*}{$\begin{array}{c}83 \\
324\end{array}$} & $82 \%$ & 181 & 269 & 315 & 352 & 531 & $88 \%$ \\
\hline RB & & & $76 \%$ & 219 & 344 & 379 & 456 & 555 & $81 \%$ \\
\hline $\mathrm{CF}$ & & & $74 \%$ & 259 & 348 & 381 & 457 & 623 & $71 \%$ \\
\hline Arcsine & & & $20 \%$ & 261 & 334 & 346 & 372 & 428 & $100 \%$ \\
\hline
\end{tabular}

